\title{
Atomic-scale intergranular crack-tip plasticity in tilt grain boundaries acting as an effective dislocation source
}

\author{
Tomotsugu Shimokawa, ${ }^{\mathrm{a},{ }^{*}}$ and Masaaki Tsuboi ${ }^{\mathrm{b}, * *}$
}

\author{
${ }^{a}$ School of Mechanical Engineering, Kanazawa University, Ishikawa 920-1192, Japan \\ ${ }^{\mathrm{b}}$ Division of Mechanical Science and Engineering, Kanazawa University, Ishikawa 920-1192, \\ Japan \\ *simokawa@se.kanazawa-u.ac.jp, +81-76-234-4692 (Corresponding auther) \\ ** tsuboi.m.882@gmail.com
}

\begin{abstract}
The intergranular fracture toughness of plastic deformable crystalline materials is strongly controlled by the plastic work ahead of the intergranular crack tip. Therefore, in studies of intergranular fracture toughness, the grain boundaries (GBs) should be regarded as both a cleavage plane and dislocation source. Combining continuum analyses and atomic simulations, this study investigates the atomic-scale mechanism of intergranular crack tip plasticity in aluminum $<112>$ tilt GBs as an effective dislocation source. To quantitatively predict the first plastic deformation near the intergranular crack tip, we first model the dislocation emission from the GBs ahead of the intergranular crack tip and analytically derive the critical stress intensity factor. If the predicted first plastic phenomenon is dislocation emission from the GBs, the resulting wedge disclination can shield the stress field near the crack. Dislocation emissions from the crack tip are accompanied by dislocation emissions from the GBs, despite the predicted difficulty of the latter. The lattice defect evolution nucleates a nanograin with a disclination at the triple-junction ahead of the crack tip, which can weaken the mechanical field near the crack tip. Consequently, when improving the intergranular fracture toughness of materials, the role of GBs as dislocation sources cannot be ignored.
\end{abstract}

Keyword: Grain boundary, Disclination, Dislocation, Interfacial fracture toughness, Molecular dynamics 


\section{Introduction}

Owing to recent progress in manufacturing technology, nanostructured materials (NSMs) can now be manufactured in bulk [1]. NSMs include ultrafine-grained (UFG) materials [2], bimodal materials [3], and nanotwined $\mathrm{Cu}$ [4]. To apply NSMs in high-strength, high-toughness structural components, we must understand their fracture phenomena [5-7]. Traditionally, fracture toughness is simply estimated from the relationship between the crack extension force and the resistance of the material [8]. In general, the former can be calculated within a continuum mechanics framework, whereas the latter is determined by physical properties such as the strength of interatomic bonding ahead of the crack tip. NSMs are characterized by a large volume fraction of grain boundary (GB) regions, the natural sites of cleavage fracture. Therefore, to understand the fracture toughness of NSMs in the above context, we must investigate the resistance of NSMs to both intragranular and intergranular cracking. Furthermore, since NSMs can plastically deform, we must consider the GB-related plastic phenomena ahead of intergranular crack tips.

First, we consider the transgranular fracture of plastic deformable materials in the absence of any GB effects. Based on the energy balance approach, crack propagation should satisfy the following condition [9-10]:

$$
G_{\mathrm{c}}=\mathcal{G}^{>} 2 \gamma_{\mathrm{s}}+\gamma_{\mathrm{p}}
$$

Here, $G$ represents the strain energy release rate corresponding to the crack extension force and $2 \gamma_{\mathrm{s}}+\gamma_{\mathrm{p}}$ represents the effective surface energy corresponding to the crack extension resistance. $\gamma_{\mathrm{s}}$ and $\gamma_{\mathrm{p}}$ represents the surface energy and the work done by plastic strain near the crack tip, respectively. A more specific description of plastic deformation that admits dislocations is provided by the stress intensity factor (SIF) approach. According to Griffith [11], ideal cleavage fracture requires that the local SIF $k\left(=K+K_{\mathrm{D}}\right)$ be larger than the critical SIF $K_{\mathrm{G}}$; that is,

$$
K_{\mathrm{c}}=K>K_{\mathrm{G}}-K_{\mathrm{D}}
$$

Here, $K$ and $K_{\mathrm{D}}$ are the SIFs caused by the applied stress and dislocations near the crack tip, respectively. $K$ and $K_{\mathrm{G}}-K_{\mathrm{D}}$ can be regarded as the crack extension force and resistance, 
respectively. This concept is the well-known dislocation shielding theory [12]. Because the critical values of $G_{\mathrm{c}}$ and $K_{\mathrm{c}}$ correspond to the fracture toughness of deformable materials, materials with high fracture toughness exhibit large plastic strain with many shielding dislocations near the crack tip. Moreover, the brittle-ductile transition behavior of materials can be determined by comparing the expansion rates of the crack expansion force and those of the plastic field.

Next, the influence of GBs on fracture toughness is incorporated by considering the intergranular fracture. In this case, a GB cleaves into two new surfaces, and the effective surface energy in Eq. (1) is modified to $\left(2 \gamma_{\mathrm{s}}-\gamma_{\mathrm{gb}}\right)+\gamma_{\mathrm{p}}$ [13], where $\gamma_{\mathrm{gb}}$ is the GB energy. Observed fracture toughness values indicate that although the first term depends on the GB structure [14], it is generally dominated by the second term $\left(\left(2 \gamma_{\mathrm{s}}-\gamma_{\mathrm{gb}}\right)<<\gamma_{\mathrm{p}}\right)[15]$; therefore, fracture toughness appears to be relatively unaltered by the GB structure if $\gamma_{\mathrm{p}}$ is a constant parameter of the material. However, fracture toughness strongly depends on GB segregation that changes the first term [16], and GB strength is strongly correlated with GB energy [17], indicating that GB structure influences the fracture toughness in practice. These results clearly show the discrepancy between the estimated and observed influence of GB structure on intergranular fracture toughness. To resolve this discrepancy, Jokl et al. expressed $\gamma_{\mathrm{p}}$ as a function of $\left(2 \gamma_{\mathrm{s}}-\gamma_{\mathrm{gb}}\right)$ [18]; specifically, they proposed that the plastic work $\gamma_{\mathrm{p}}$ caused by dislocations emitted from the crack tip increases with the ideal cleavage fracture resistance $\left(2 \gamma_{\mathrm{s}}\right.$ $-\gamma_{\mathrm{gb}}$ ). Yamaguchi et al. have explained the strong dependence of the fracture toughness on the type and coverage of segregated solute observed by the experiments with a thermodynamic approach aided by first-principles calculations [19].

Apart from forming a cleavage plane, do GBs play other important roles in the transgranular fracture of NSMs? Recently, a dislocation source role has been identified for GBs, providing clues into the unique mechanical properties of NSMs [20-23]. For example, the fracture toughness of UFG metals is improved at low temperatures [24,25]. This phenomenon may be explained by dislocation emission from GBs near the transgranular crack tip [26]. Furthermore, atomic simulation studies have elucidated the mechanism of dislocation emission from GBs and its dependence on GB structure [27-29]. These studies have sparked an interest in GBs as a dislocation source and their effect on intergranular fracture toughness. Because dislocations emitted from GBs contribute to the plastic work $\gamma_{\mathrm{p}}$ and their 
characteristics greatly affect the $K_{\mathrm{D}}$, the relationship between the intergranular fracture toughness and GB structures must be reconsidered from the perspective of GBs as a dislocation source. Such a reinterpretation is essential for the material design of NSMs based on GB engineering [30].

In this study, we investigate the atomic-scale intergranular crack tip plasticity in tilt GBs acting as an effective dislocation source. Our investigation combines continuum analyses and atomic simulations. In Section 2, after briefly introducing the GB properties used in the atomic simulations, we model the dislocation emission from GBs ahead of the intergranular crack tip and analytically derive the critical SIF. From the critical SIF, we quantitatively predict the first plastic deformation phenomenon near the intergranular crack tip. Section 3 examines the intergranular crack tip plasticity in atomic simulations and investigates how the microstructures caused by dislocation emission from GBs affect the fracture toughness. The investigated microstructures are a wedge disclination [31] at the GB and a nanograin with a disclination at the triple junction near the crack tip. Discussions and conclusions are presented in Sections 4 and 5, respectively.

\section{Theories and Computational Methods}

In this study, we use the theory of isotropic linear elasticity to derive the critical SIF to dislocation emission from GBs, and predict the first plastic deformation near the intergranular crack tip in atomic models. Therefore, the analyzed material is aluminum (Al), which can be considered as an isotropic elastic body. In the atomic simulations, we adopt the embedded atom method (EAM) proposed by Mishin [32]. The anisotropic factor of the atomic potential, calculated as $c_{44} /\left(c_{11}-c_{12}\right)$, is 1.25 .

\subsection{Grain boundary structure}

The intergranular crack tip plasticity is investigated in four Al symmetrical $<112>$ tilt GBs: $\Sigma 175, \Sigma 73, \Sigma 77$, and $\Sigma 15$. These GBs exist close to the stable $\Sigma 11 \mathrm{~GB}$ and easily emit dislocations under tensile loading applied perpendicular to their planes [29]. The structural and physical properties of the GBs are summarized in Table 1. The detailed atomic structures and the dislocation emission mechanism are briefly described below. 
Among the GBs close to the analyzed GBs, the most stable is $\Sigma 11 \mathrm{~GB}$ that misorientation angle $\theta_{\mathrm{GB}}=62.96^{\circ}$. The structure of $\Sigma 11 \mathrm{~GB}$ can be represented by structural units (SUs; yellow symbols in Fig. 1(a)). This SU is called a $B$-SU [29]. The colors in Fig. 1 denote the stacked atomic layers at different depths along the $<112>$ direction. Note that the GB has a six-layer periodicity. In terms of the depth of atoms constituting the $B$-SU, the periodic structure of $\Sigma 11 \mathrm{~GB}$ is $\left|B^{1} B^{2} B^{3} B^{4} B^{5} B^{6}\right|$. However, because the results of this study were independent of atomic depth, the depth information was ignored when determining the GB structure with SUs. To represent the GB structures of $\Sigma 175, \Sigma 73, \Sigma 77$, and $\Sigma 15$, we regularly introduce SUs into $\Sigma 11 \mathrm{~GB}$, as shown in Fig. 1(b-d). This SU is called $C$-SU. The density of $C$-SU and the GB energy increase with increasing deviation of the misorientation angle $\Delta \theta$ from $\Sigma 11 \mathrm{~GB}$, as shown in Fig. 1 and Table 1. Setting $\Sigma 11 \mathrm{~GB}$ as a reference structure, we can consider $C$-SU to be a GB dislocation that forms the deviation from $\Sigma 11$ GB [29].

When these GBs are deformed under tensile loading applied perpendicular to their planes, the GB dislocation ( $\kappa)$ in $C$-SU decomposes into a lattice edge dislocation $(\zeta)$ emitted into a grain and glissile GB dislocation $\left(\zeta^{\prime \prime}\right)$ with a Burgers vector parallel to the GB plane: $\boldsymbol{b}^{\kappa}=\boldsymbol{b}^{\zeta}+$

$\boldsymbol{b}^{\zeta^{\prime \prime}}\left(\right.$ see Fig. 3(b)), e.g., $\boldsymbol{b}^{\kappa}=\frac{2}{11}[\overline{3} 1 \overline{1}], \boldsymbol{b}^{\zeta}=\frac{1}{2}[\overline{1} 10]$, and $\boldsymbol{b}^{\zeta^{\prime \prime}}=\frac{1}{22}[\overline{1} \overline{\overline{7}} \overline{4}]$ in this study. Since this type of decomposition rarely occurs under compressive loading, dislocation generation by GBs is asymmetric. This asymmetry arises from the relationship between the Burgers vector components of the lattice dislocation $\zeta$ and the GB dislocation $\kappa$. Because dislocation emission transforms $C$-SU to $B$-SU, it structurally transforms the original GB into $\Sigma 11 \mathrm{~GB}$. The tensile stress required for GB dislocation emission decreases with increasing spacing $h$ of the $C$-SUs [29]; hence, dislocations are most easily emitted from $\Sigma 175$ GB in this study.

\subsection{Atomic simulation setups}

Figure 2 (a) is a schematic of the analysis model used in the atomic simulations. In all models, initial $L_{y}$ and $L_{z}$ are set to $1.5 \mathrm{~nm}$ and $90 \mathrm{~nm}$, respectively, while $L_{x}$ is varied. The dimensions of $L_{x}$ are listed in Table 1. A periodic boundary condition is imposed in the $y$-direction. The tilt GB is constructed by two grains (1 and 2) arranged in the center of the model. Because the plastic deformation phenomena near the crack tip are sensitive to the direction of crack propagation [33-35], an intergranular crack of length $L_{\mathrm{c}}=20 \mathrm{~nm}$ is introduced to the GB from the right or left free surface. Möller et al. report that even when no 
crack tip plasticity is involved, the intergranular fracture toughness depends on the exact position of the crack tip within the SUs of the GB [35]. Therefore, in our analysis model, we vary the distance $\alpha_{(i)}$ between the crack tip and the first $C$-SU to investigate its influence on the plastic phenomena near the crack tip. Here, the $i$ in parentheses represents the SU number counted from the crack tip. Figure 2 (b-d) shows the atomic configurations near the left crack tip in the $\Sigma 175$ GB model. Because $\Sigma 175$ GB has $|(10 B) C|$ periodicity, we construct 11 analysis models, varying $\alpha_{(i)}$ from $\alpha_{(1)}$ to $\alpha_{(11)}$. Right crack models are prepared in the same manner (Fig. 2(e)). Since there are four types of GBs, we construct a total of 52 analysis models.

Each analysis model is relaxed at $100 \mathrm{~K}$ and subjected to tensile deformation tests in the $z$-direction. A simple tensile boundary condition is adopted to all deformation tests instead of rigid boundary conditions with a displacement control (DC) layer surrounding a crack tip that displacement field is given by linear elastic fracture mechanics, because we do not want to consider a configuration force on the crack tip that increases as it moves nearer the rigid boundary. In this study, tensile deformation tests are performed by controlling the 0.6-nm-wide DC regions shown in Fig. 2. The atomic displacements in the $x$ - and $y$-directions of the DC regions are not controlled. The strain rate is $1 \times 10^{8} \mathrm{~s}^{-1}$ and the temperature is maintained at $100 \mathrm{~K}$ by the velocity scaling method [36]. To mimic the plane strain condition, $L_{y}$ is fixed during deformation. The local face-centered-cubic (fcc) and hexagonal-close-packed (hcp) crystal structures as well as the defect atomic structures are classified by a common neighbor analysis (CNA) [37]. Because the analysis models are geometrically constricted in the $y$-direction, the only active slip systems in Grains 1 and 2 are $(111)_{1}[1 \overline{1} 0]_{1}$ and $(111)_{2}[1 \overline{1} 0]_{2}$, respectively (see Fig. 2(a)). However, by limiting the active slip systems, we can more easily compare the intergranular crack tip plasticity observed in the atomic models with that predicted by continuum SIF analyses.

As shown in Fig. 2 (b-e), the crack tip in the atomic models is wedge-shaped rather than infinitely sharp; hence, the crack tip position is corrected in the continuum analyses. The atomic planes of the left and right wedge-shaped crack tip are (111) and (110), respectively, and the crack surfaces in the equilibrium state are separated by approximately $1.2 \mathrm{~nm}$. If the wedge crack tip is regarded as a crack with a finite tip radius $\rho(\approx 0.6 \mathrm{~nm})$, it shifts a distance of $\rho / 2$ behind the physical tip [38]. Note that all $\alpha$ are added $\rho / 2$ in this study. 


\subsection{Stress intensity factor to dislocation emission from grain boundary ahead of intergranular crack tip}

Two well-established and widely used parameters are the critical SIFs that induce ideal intergranular cleavage fracture [13] and dislocation emission from the crack tip [12, 39]. However, the critical SIF to dislocation emission from GBs ahead of the intergranular crack tip are yet to be derived, despite the significant effect of this phenomenon on crack tip plasticity [34]. This section derives the critical SIF to dislocation emission from the tilt GBs using an improved Rice-Thomson model [12].

Rice and Thomson [12] determined the critical SIF to dislocation emission from the crack tip by imposing a force $f$ on a dislocation at some positive cutoff distance $r_{0}$ from the crack tip. Provided that no other dislocations exist near the crack tip, the total force $f$ on the dislocation is the sum of the direct crack-dislocation force $f_{\mathrm{K}}$ caused by the applied SIF $K_{\mathrm{I}}$, the self-image force $f_{\mathrm{d}^{2}}$ caused by the free surface effect, and the surface-step force $f_{\text {se }}$ caused by the nucleation of the surface step, which increases the surface energy. Because the dislocation core is treated as a singularity in continuum mechanics, $f$ is rendered large and negative by $f_{\mathrm{d}^{2}}$ when the dislocation is very close to the crack tip, and dislocations are never emitted from the crack tip. To overcome this problem, Rice and Thomson specified a cutoff distance $r_{0}$ corresponding to the magnitude of the dislocation core size, and assumed that the crack tip can emit a dislocation if $f$ is positive outside of $r_{0}$.

Based on the above model, we derived the critical SIF to dislocation emission from a site of tilt GBs at distance $\alpha$ from the crack tip. The analysis model is shown in Fig. 3. If the force acting on the dislocation $\zeta$ at distance $r$ from the grain boundary satisfies the following relationship, we assume that the dislocation $\zeta$ can be emitted from the GB site.

$$
f=f_{\mathrm{K}}+f_{\mathrm{d}^{2}}+f_{\mathrm{dd}^{\prime}}+f_{\mathrm{gbe}}>0 \quad\left(r=r_{0}\right)
$$

Our model differs from the Rice-Thomson model in two ways. First, we introduce a GB transformation force $f_{\text {gbe }}$ that accounts for the transformation of the original local GB structure (GB1) into another GB structure (GB0) after emitting dislocation $\zeta$, as shown in Fig. 3(b). Second, the emission of dislocation $\zeta$ is followed by a residual dislocation $\zeta^{\prime}$, inducing a dislocation-dislocation interaction force $f_{\mathrm{dd}^{\prime}}$, as shown in Fig. 3 (a). $f_{\mathrm{K}}$ and $f_{\mathrm{dd}^{\prime}}$ are calculated 
conventionally (see Appendix A). The newly introduced $f_{\text {gbe }}$ is detailed below.

We suppose that $f_{\text {gbe }}$ is caused by the local GB structural transformation from GB1 to GB0 after dislocation emission from the GB. The energy change $\Delta E_{\mathrm{gb}}$ due to the dislocation emission is given by

$$
\Delta E_{\mathrm{gb}}=\Delta \gamma_{\mathrm{gb}} h
$$

Here, $\Delta \gamma_{\mathrm{gb}}$ is the GB energy difference between GB1 and GB0, and $h$ is the spacing between GB dislocations $\kappa$. Since the energy change $\Delta E_{\mathrm{gb}}$ must influence the dislocation emission from the GB, we suppose that $f_{\text {gbe }}$ can be calculated analogously to $f_{\text {se }}[12]$ as follows.

$$
f_{\mathrm{gbe}}=-\frac{4 \Delta E_{\mathrm{gb}} r_{0}}{\pi\left(r_{0}^{2}+r^{2}\right)}
$$

$\Delta E_{\mathrm{gb}}$ is then obtained by integrating $-f_{\mathrm{gbe}}$ from $r=0$ to $r_{0}$. The GB dislocation $\kappa$ is decomposed into a lattice dislocation $\zeta$ and grain boundary dislocation $\zeta^{\prime \prime}$ with Burgers vector parallel to the GB plane (see Fig. 3(b)). However, we ignore the influence of $\zeta^{\prime \prime}$ on $\Delta E_{\mathrm{gb}}$ in this study because $\zeta$ " does not contribute the misorientation angle change of GBs and the residual dislocation effect on $f$ is already considered in $f_{\mathrm{dd}^{\prime}}($ see Appendix $\mathrm{A})$.

The remaining parameter to be determined is the cutoff distance $r_{0}$. In this study, we determine $r_{0}$ by comparing the two critical SIFs to dislocation emission from the crack tip: the Rice and Thomson approach made use of elasticity solutions for a fully formed dislocation [12] and the Rice approach based on the Peierls concept [39], as follows.

$$
r_{0}=\frac{1-v}{2 \pi \mu \gamma_{\mathrm{us}}}\left(\frac{\mu b}{2 \sqrt{2}(1-v)}+2 \sqrt{2} \gamma_{\mathrm{s}}\right)^{2} \frac{1}{1+(1-v) \tan ^{2} \chi}
$$

Here, $\gamma_{\text {us }}$ and $\chi$ denote the unstable stacking fault energy and the angle of the slip direction on the slip plane, respectively and both are required to determine the critical SIF of Rice model [39]. In terms of the EAM interatomic potential of Mishin [32], we have for Al: $b=0.286 \mathrm{~nm}$, $\gamma_{\mathrm{us}}=0.188 \mathrm{~J} / \mathrm{m}^{2}$, and $\gamma_{\mathrm{s}}=\gamma_{\mathrm{s}(111)}=0.87 \mathrm{~J} / \mathrm{m}^{2}$. Estimating the shear modulus $\mu$ and Poisson's ratio $v$ by the Voigt approximation [40], we obtain $\mu=29.3 \mathrm{GPa}$ and $v=0.33$. When $\chi=30^{\circ}$, corresponding to the leading partial dislocation of a perfect edge dislocation, we obtain $r_{0}=$ $2.62 b=0.75 \mathrm{~nm}$. This value is consistent with the width of the extended edge dislocation of Al expressed by the EAM potential, estimated as $0.7 \mathrm{~nm}$ by linear elastic theory [41]. 
Consequently, the influence of $\gamma_{\text {us }}$ on the dislocation emission phenomena is considered through $r_{0}$ in our model.

In Eq. (3), $f_{\mathrm{K}}$ alone is a function of applied stress; hence, the critical SIF to dislocation emission from GBs, denoted $K_{\mathrm{disl}, \mathrm{gb}}^{\mathrm{Th}}$, can be determined as the $K_{\mathrm{I}}$ that satisfies the condition of Eq. (3).

\section{Results}

\subsection{Initial plastic deformation phenomena around intergranular crack tip}

In this section, we investigate the influence of the crack propagation direction on the first plastic phenomenon near the crack tip and compare the first plastic phenomena in atomic simulations with that predicted by the continuum analyses. The critical SIF to dislocation emission from the crack tip $K_{\text {disl,ck }}^{\mathrm{Th}}$ is calculated by the Rice-Thomson model [12] and that to ideal intergranular cleave fracture $K_{\mathrm{cleav}}^{\mathrm{Th}}$ is estimated by $\sqrt{2 \mu\left(2 \gamma_{\mathrm{s}}-\gamma_{\mathrm{gb}}\right) /(1-v)}$ [13]. The Rice model [39] also gives the same values of $K_{\text {disl,ck }}^{\mathrm{Th}}$ because $r_{0}$ is determined by Eq. (6). The $K_{\text {disl,ck }}^{\mathrm{Th}}$ and $K_{\text {cleav }}^{\mathrm{Th}}$ for each GB are listed in Table 1. Because of the simple tensile boundary condition used in this study, crack tip stress fields of atomic models show a little different from that of $K_{\mathrm{I}}$ (see Fig. B.1). By correlating with $K_{\mathrm{I}}$, we obtained the stress field near the crack tip in atomic models as $F_{1} F_{2} K_{\mathrm{I}}$. Here, $F_{1}$ and $F_{2}$ are the geometrical correlation factor due to the geometry of finite size specimens and an additional correlation factor due to the simple tensile boundary condition, respectively. In calculating the critical SIF for the atomic models, we decided that the critical state of the dislocation emission from a crack tip or GBs was reached when the leading position of the emitting dislocation, $r_{\mathrm{d}}$, was $1.5 \mathrm{~nm}$ from the source. Detailed explanations of $F_{2}$ and $r_{\mathrm{d}}$ are provided in Appendices B and C, respectively.

\subsubsection{Left crack}

Figure 4 plots the $K_{\text {disl,gb }}^{\mathrm{Th}}$ of the left cracks as a function of $\alpha$ in broken lines. $K_{\text {disl,ck }}^{\mathrm{Th}}$ is omitted from Fig. 4 because all $K_{\text {disl,ck }}^{\text {Th }}$ values exceed $0.6 \mathrm{MPam}^{1 / 2}$ (see Table 1). The analytical model predicts that $K_{\text {disl,gb }}^{\mathrm{Th}}$ increases with $\alpha$. The atomic simulation results of 
$K_{\text {disl,gb }}^{\mathrm{MD}}$ are also plotted in Fig. 4. According to the atomic models, the first plastic deformation in any left crack is dislocation emission from $C$-SU in the GBs. Remarkably, our purely analytical $K_{\text {disl,gb }}^{\mathrm{Th}}$ strongly correlates with $K_{\text {disl,gb }}^{\mathrm{MD}}$ although $K_{\text {disl,gb }}^{\mathrm{Th}}$ overestimates $K_{\text {disl,gb }}^{\mathrm{MD}}$ at large $\alpha$. The newly introduced term in the Rice-Thomson model [12], $f_{\text {gbe }}$ in Eq. (5), alone includes the GB information and there is a possibility that the actual functional shape of $f_{\mathrm{gbe}}$ is not completely the same as $f_{\text {se }}$; therefore, we attempt to fit $K_{\text {disl,gb }}^{\mathrm{Th}}$ to $K_{\text {disl,gb }}^{\mathrm{MD}}$ by simply scaling $f_{\text {gbe. }}$ The solid lines in Fig. 4 show the $K_{\text {disl,gb }}^{\mathrm{Th}}$ values at $1.15 f_{\text {gbe }}$. Since $f_{\text {gbe }}$ is a linear function of the $C$-SU spacing $h, K_{\mathrm{disl}, \mathrm{gb}}^{\mathrm{Th}}$ of GB structures with larger $h(\Sigma 175$ and $\Sigma 73)$ are largely influenced by this modification. This slight modification of $K_{\mathrm{disl}, \mathrm{gb}}^{\mathrm{Th}}$ provides more realistic $K_{\text {disl,gb }}^{\mathrm{MD}}$ values than the as-derived $K_{\text {disl,gb }}^{\mathrm{Th}}$, indicating that our modeling of dislocation emission from GBs can reasonably predict the atomic scale intergranular crack tip plasticity resulting from the GB characteristics. Note that $K_{\text {cleav }}^{\mathrm{Th}}$ is smaller than $K_{\text {disl,gb }}^{\mathrm{Th}}$ for small $\alpha$. However, ideal cleavage fracture does not occur in atomic models for reasons discussed in Section 4.

Next, we examine the detailed phenomena of dislocation emission from GBs ahead of the left crack tip. Depending on $\alpha$, three typical phenomena are observed. Figure 5 shows the typical atomic configurations near the right crack tips of $\Sigma 77$ with $\alpha_{(2)}=0.9 \mathrm{~nm}, \Sigma 73$ with $\alpha_{(4)}=$ $1.4 \mathrm{~nm}$, and $\Sigma 175$ with $\alpha_{(11)}=3.4 \mathrm{~nm}$. The critical states of each model are shown in Fig. 5 (b), (c), and (e). The first state occurs at small $\alpha: \alpha_{(1)}$ and $\alpha_{(2)}$. In this case, the dislocation emission occurs not from the nearest $C$-SU but from the second nearest $C$-SU at distance $\alpha+h$, as shown in Fig. 5 (a) and (b). This displacement probably results from atomic structural change at the crack tip starting from the nearest $C$-SU, as shown in Fig. 5(b). Therefore, all $K_{\text {disl,gb }}^{\mathrm{MD}}$ values of the atomic models with $\alpha_{(1)}$ and $\alpha_{(2)}$ in Fig. 4 are plotted at $\alpha_{(1)}+h$ and $\alpha_{(2)}+h$. The second state, occurring at intermediate $\alpha$, is the emitted dislocation trapping near the crack tip due to the strong elastic interaction (see Fig. 5 (c) and (d)). As noted later (subsection 3.2.1), successive dislocations can be continuously emitted into grains from the second and third $C$-SUs under further applied loading. The final state is pure dislocation emission from the GB when $\alpha$ exceeds $3 \mathrm{~nm}$, which produces a noticeable step of the GB plane after the emission of 
the trailing partial dislocation. This step corresponds to the GB dislocation with Burgers vector parallel to the boundary plane [29] (see dislocation $\zeta^{\prime \prime}$ in Fig. 3(b)).

\subsubsection{Right crack}

Figure 6 plots the $K_{\text {disl,ck }}^{\mathrm{Th}}$ and modified $K_{\mathrm{disl}, \mathrm{gb}}^{\mathrm{Th}}$ described in 3.1.1 of the right cracks as functions of $\alpha$. Comparing $K_{\mathrm{disl}, \mathrm{Tk}}^{\mathrm{Th}}$ and $K_{\mathrm{disl}, \mathrm{gb}}^{\mathrm{Th}}$ we find that in most cases, dislocation is more easily emitted from the crack tip than from the GBs. The exception is $\Sigma 175 \mathrm{~GB}$, with $\alpha$ smaller than $0.6 \mathrm{~nm}$. The atomic simulation results are also plotted in Fig. 6 . According to the $K_{\text {disl,ck }}^{\mathrm{Th}}$ and $K_{\mathrm{disl}, \mathrm{gb}}^{\mathrm{Th}}$, dislocation emission from GBs in the atomic models is observable only for $\Sigma 175$ GB with $\alpha_{(1)}=0.58 \mathrm{~nm}$. In the other cases, the first dislocation in the atomic models is emitted from the crack tip. Consequently, the analytical model well expresses the first plastic deformation observed in the atomic models. Note that although $K_{\text {cleav }}^{\mathrm{Th}}$ is slightly smaller than $K_{\text {disl,ck }}^{\mathrm{Th}}$ in $\Sigma 73, \Sigma 77$, and $\Sigma 15$ GBs (see Table 1), the first dislocation is emitted from the crack tip without cleavage fracture, for reasons to be discussed in Section 4.

While $K_{\text {disl,ck }}^{\mathrm{Th}}$ is constant with respect to $\alpha, K_{\text {disl,ck }}^{\mathrm{MD}}$ is a decreasing function of $\alpha$, as shown in Fig. 6 . In the case of $\Sigma 11 \mathrm{~GB}$ without $C$-SU, we find that $K_{\text {disl,ck }}^{\mathrm{Th}}\left(\approx 0.38 \mathrm{MPam}^{1 / 2}\right)$ and $K_{\text {disl,ck }}^{\mathrm{MD}}\left(\approx 0.39 \mathrm{MPam}^{1 / 2}\right)$ are almost identical. Thus, we can suppose that the $\alpha$ dependence of $K_{\text {disl,ck }}^{\mathrm{MD}}$ manifests from the mechanical field imposed by the GB dislocation in $C$-SU. Figure 7 (a) and (b) shows the $\sigma_{z}$ stress distribution near the right crack tip of $\Sigma 175 \mathrm{~GB}$ under zero applied loading. A clear stress field appears around each $C$-SU, and the plus or minus sign of the stress field is consistent with the analytical solution [41] of the GB dislocation in $C$-SU (Fig. 1). The mechanical field at the crack tip changes as $\alpha$ changes. Figure 7 (d) plots the average stress $\sigma_{z \text {,tip }}$ in the crack tip region $S$ shown in Fig. 7 (c) as a function of $\alpha$. At each GB, $\sigma_{z}$,tip increases from negative to positive as $\alpha$ increases; therefore, the mechanical field near the crack tip can be shielded (anti-shielded) by a $C$-SU that is close to (distant from) the crack tip. This tendency can explain the results of Fig. 6. Consequently, the local mechanical field introduced by the GB structures influences the dislocation emission phenomenon from the crack tip, identically to cleavage fracture without crack tip plasticity [35]. 


\subsection{Effect of dislocation emission from grain boundaries on intergranular crack tip plasticity}

In this section, we focus on the plastic phenomena near the crack tip and investigate how GBs acting as dislocation sources influence the intergranular fracture toughness.

\subsubsection{Left crack}

Because the tips of left cracks do not readily emit dislocations (see Table 1), they should undergo brittle cleavage fracture (according to conventional theories that ignore the dislocation emission from GBs). However, intergranular fracture toughness can be improved by the following two mechanisms related to the dislocation emission from GBs.

The first mechanism is the transformation of a GB into a more stable GB structure. Figure $8(\mathrm{a}-\mathrm{c})$ shows the lattice defect evolution of $\Sigma 15 \mathrm{~GB}$ with $\alpha_{(2)}=0.9 \mathrm{~nm}$ in $K_{\mathrm{I}}=0.51 \mathrm{MPam}^{1 / 2}$, $0.67 \mathrm{MPam}^{1 / 2}$, and $0.78 \mathrm{MPam}^{1 / 2}$, respectively. The colors of the atoms represent their $\sigma_{z}$ stress components. As mentioned in 3.1.1, the first dislocation is emitted from a $C$-SU. Successive dislocations are continuously emitted from the $C$-SUs ahead of the left crack tip, as shown in Fig. 8 (b) and (c). $\Sigma 15$ GB then transforms into stable $\Sigma 11$ GB. The other GBs in this study undergo the same transformation, indicating that $K_{\text {cleav }}^{\text {Th }}$ can be improved by the GB transformation induced by dislocation emission from GBs as shown in Table 1.

The second mechanism is the disclination shielding effect. As shown in Fig. 8 (a), before the first dislocation emission from the GB, the stresses are concentrated near the left crack tip. After the first dislocation emission, an apparent positive stress field develops at the border of the $\Sigma 15$ and $\Sigma 11$ GBs while the stress concentration at the crack tip begins to weaken despite $K_{\mathrm{I}}$ increases. Therefore, the mechanical field evolves identically to crack propagation along the GB. Because the misorientation angles of $\Sigma 15$ and $\Sigma 11$ GBs are different, a wedge disclination dipole with a Frank vector $\omega_{1}=\theta_{\Sigma 15}-\theta_{\Sigma 11}$ nucleates after the dislocation emission (see Fig. 9 (a)). The positive disclination interacts with the crack surface and may easily disappears. The values of $\omega_{1}$ for all GB models are listed in Table 2, and the $\sigma_{z}$ distributions of all GBs with the same nucleated disclination positions are plotted in Fig. 10. We observe that the magnitude of the mechanical field around the disclination increases with $\omega_{1}$ while the stress concentration at the crack tip of $\Sigma 15$ shown in Fig. 10 (d) is obviously weaker than that of $\Sigma 175$ shown in Fig. 
10(a). The SIF caused by the disclination dipole shown in Fig. 9 (a) can be calculated from the $K_{\mathrm{D}}$, regarding the disclination dipole as a continuous distribution of edge dislocations [42]. Specifically, we have

$$
k_{\text {Discli }}=-\frac{2 \mu \omega \sqrt{R}}{(1-v) \sqrt{2 \pi}}
$$

where $R$ is the dipole arm length. According to Eq. (7), the shielding effect of the disclination dipole increases with $\omega$ and $R$; therefore, we conclude that the mechanical field of the crack tip can be shielded by nucleation of the negative wedge disclination at the border of the original and $\Sigma 11 \mathrm{GBs}$, itself induced by dislocation emission from the original GB. It is interesting to investigate the atomic structure of the disclination core, but all disclinations observed in this study are not perfect disclinations but partial disclination with GBs [31] that structure can be expressed by the combination of SUs as shown in Fig. 8(d). Therefore, the identification of the partial disclination core structure on GBs is hard although we can observe the mechanical field of the partial disclination centered around the border of GBs with different misorientation angles.

Together, the stabilization of GB structures and the disclination shielding effect increase the intergranular fracture toughness of left cracks. Furthermore, despite the difficulty of dislocation emission from the crack tip, the expansion rate of the plastic field ( $\dot{\gamma}_{\mathrm{p}}$ and $\left.\dot{K}_{\mathrm{D}}\right)$ is maintained because the GBs act as dislocation sources, ensuring ductile behavior of the materials.

\subsubsection{Right crack}

In most cases, the first plastic phenomenon near the right intergranular crack tip is the dislocation emission from the crack tip (see Fig. 6). If this phenomenon is continuous, as predicted by the analytical models and presumed by Jolk et al. [18], the intergranular crack tip can behave in a ductile manner, similar to the ductility ensured by the dislocation shielding effect [12]. However, as $h$ increases in the atomic models, the microstructure is nucleated ahead of the intragranular crack tip by dislocation emission from the GB, as shown in Fig. 11 (a) and (b). Furthermore, as $h$ reduces, crack propagation is accompanied by dislocation 
emission from the crack tip. We now explain the two mechanisms of crack tip plasticity and investigate how the microstructure influences the mechanical field near the crack tip.

We first consider the GBs with larger $h(\Sigma 77, \Sigma 73$, and $\Sigma 175)$. Figure 11 (a) and (b) shows the atomic configurations near the right crack tip in the $\Sigma 77$ and $\Sigma 73$ models, respectively, under tensile strain $\varepsilon_{z} \approx 0.04$. As predicted by the analytical model, continuous dislocation emission in one direction from the crack tip leads to crack blunting. Unexpectedly, the first GB dislocation emission occurs during the third (fourth) dislocation emission from the right crack tip of $\Sigma 77$ ( $\Sigma 73) \mathrm{GB}$, and the number of dislocations emitted from the GB increases with increasing crack tip opening displacement (CTOD) caused by dislocation emission from the crack tip. Therefore, the unpredictable GB dislocation emission near the right crack tips based on Eq. (3) could be induced by the crack blunting. As discussed for the left crack tips in the preceding subsection, the GB structure becomes a stable $\Sigma 11 \mathrm{~GB}$, with consequent increase of the intergranular fracture toughness. Furthermore, the dislocations emitted from the GB compose a low-angle GB (LAGB) near the crack tip. This LAGB is terminated by the crack tip and triple junction among the original GB, $\Sigma 11$, and the LAGB; hence, a new nanograin nucleates near the crack tip. We now ponder the question: what is the role of the nanograin in fracture toughness?

Ovid'ko et al. [43] reported similar microstructural evolution of nanoscale grain nucleates, which they modeled as disclination quadruples. They analytically investigated nanograin nucleation caused by splitting and migration of grain boundaries, and confirmed that nanograin nucleation effectively enhances the toughness of nanocrystalline ceramic [43]. Here, we investigate the influence of the nanograin on plastic deformation near the crack tip in our atomic models. Identically to the left crack tip shown in Fig. 8, a disclination dipole of strength $\omega_{1}$ is generated by the dislocation emission from the GBs, but the $\omega_{1} \mathrm{~s}$ developed in the right and left cracks are of opposite sign (see Fig. 9). Therefore, according to Eq. (7), this disclination dipole anti-shields the right crack tip. On the other hand, the terminated LAGB with misorientation angle $\theta_{\mathrm{L}}$ can be regarded as a disclination dipole with $\omega_{2}=\theta_{\mathrm{L}}$ (see Fig. 9 (b)). Here, $\theta_{\mathrm{L}}$ is equal to $2 \sin ^{-1} b / 2 d$ [13], where $d$ represents the spacing between the edge dislocations. Therefore, positive and negative disclinations of respective strengths $\omega_{1}$ and $-\omega_{2}$ appear at the triple junction. The $\omega_{1}$ and $\omega_{2}$ values for each GB are listed in Table 2. Because the total Frank vector $\left(\omega_{3}=\omega_{1}-\omega_{2}\right)$ is always negative, the microstructure could shield the 
right crack tip analogously to the left crack based on Eq. (7). Negative disclination nucleation at the triple junction is confirmed in the stress distributions generated by the atomic models. Figure 11(c-e) shows the $\sigma_{z}$ distributions near the right crack tip of each $\Sigma$ GB at $\varepsilon_{z} \approx 0.04$. Tensile stress fields appear not at the intergranular crack tip of $\Sigma 11$ but at the triple junctions, and their magnitude increases with increasing magnitude of $\omega_{3}$. Because the growing nanograin more-or-less retains its shape to maintain an energetically stable symmetrical GB plane (see Figs. 9(b) and 11(a), (b)), $R$ linearly increases with increasing CTOD, which is caused by dislocation emission from the crack tip. Therefore, nanograin nucleation ahead of the crack tip induced by dislocation emission from the GB can improve the intergranular

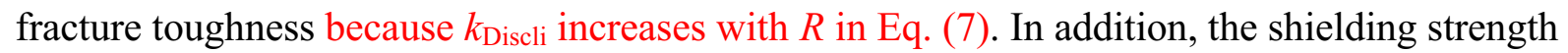
increases with $\omega_{3}$, which is related to the GB structures, and by the $\gamma_{\mathrm{p}}$ imposed by dislocation emission from the intragranular crack tip. As can be observed in Fig. 11 (a) and (b), the dislocation immediately emitted from the crack tip is trapped by the LAGB, and fewer dislocations are emitted in $\Sigma 77$ GB than in $\Sigma 73$ GB. Consequently, the CTODs differ among the GBs even under the same tensile strain $\left(\varepsilon_{z} \approx 0.004\right)$. Hence, the expansion rate of plastic field $\dot{\gamma}_{\mathrm{p}}$ decreases with $d$ ( or $h$ ) not only because of back stress caused by the previous emitted dislocations, but also because of nanograin nucleation.

However, such microstructures are absent in GBs with smaller $h(\Sigma 15)$. Figure 12 shows the atomic configurations around the left crack tip of the $\Sigma 15 \mathrm{~GB}$ with $\alpha_{(3)}=1.2 \mathrm{~nm}$. As the dislocation emits from the crack tip region, the right crack propagates along the $\Sigma 15 \mathrm{~GB}$, disordering the crack tip structure, as shown in Fig. 12 (b-d). The disordered structure could be caused by the high density of $C$-SU at the $\Sigma 15 \mathrm{~GB}$; the free volume and energy are higher in $C$-SU than in stable $B$-SU [29]. Dislocations are continuously emitted from the disordered region of the crack tip; these slip planes are unrelated to the $C$-SU shown in Fig. 12 (a). Because the emitted dislocations affect the shielding of the mechanical fields of the crack tips, anti-shielding dislocations remain in disordered GBs. Ahead of the intergranular crack tip, the anti-shielding field drives the crack propagation. This phenomenon is also confirmed in other $\Sigma 15$ GBs with different $\alpha$. Since the disclination strength $\omega_{3}$ at the triple junction increases with decreasing $h$, the $\omega_{3}$ in the $\Sigma 15$ GB models is probably too large to decompose the GB into $\Sigma 11 \mathrm{~GB}$ and LAGB. This result implies that a threshold $\omega_{\mathrm{c}}$ exists at the triple junction, 
above which GBs cannot maintain stable disclination.

Consequently, the larger the Frank vector $\omega_{3}$ of a disclination at the triple junction of a nucleated nanograin and the bigger the grown nanograin, the higher the increase in fracture toughness. However, if $\omega_{3}$ exceeds the critical value $\omega_{\mathrm{c}}$, crack propagation accompanies the dislocation emission from the crack tip region. Zhou et al. conducted tensile deformation tests in atomic models, and similarly reported a critical Frank vector $\omega_{\mathrm{c}}$, which controls the fracture mechanisms of bicrystals containing a negative wedge disclination at the center of the GB [44]. Therefore, by controlling the character and strength of disclinations near the crack tip caused by lattice defect evolution, which closely relates to the role of GBs as dislocation sources, we can obtain materials with excellent strength and ductility.

\section{Discussion}

We first consider the causes of the discrepancy between the predicted and observed first plastic deformation near the intergranular crack tip. As mentioned in 3.1, the first plastic deformation in all atomic models is a dislocation, emitted either from the crack tip or from the GB. However, in some of the analytical models, such as the $\Sigma 73, \Sigma 77$, and $\Sigma 15$ GBs in the left cracks and all GBs with large $\alpha$ in the right cracks, the first plastic phenomenon is ideal intergranular cleavage fracture. This discrepancy might arise from two sources. First, the $C$-SU contribution to the mechanical field of the crack tip is ignored in the analytical models of $K_{\text {cleav }}^{\mathrm{Th}}$ and $K_{\text {disl,ck }}^{\mathrm{Th}}$ (see subsection 3.1.2); second, the atoms are structurally rearranged ahead of the crack tip. In atomic models, the atomic structure at the crack tip is disordered by atomic bonding recombination, as shown in Fig. 5 (b). Möller reported that bond trapping of GB cracks improves the GB fracture toughness [35]. Consequently, the actual critical SIF for

crack cleavage is larger than the ideal cleavage fracture $K_{\text {cleav }}^{\text {Th }}$ because extra work is required for recombining the atomic bonds near the crack tip. When $C$-SU is close to the crack tip, recombination is favored because the free volume of $C$-SU exceeds that of $B$-SU.

We next consider the relationship between fracture toughness and $\left(2 \gamma_{\mathrm{s}}-\gamma_{\mathrm{gb}}\right)$. As mentioned in the Introduction, Jokl et al. presumed that the plastic work $\left(\gamma_{\mathrm{p}}\right)$ is an increasing function of $\left(2 \gamma_{\mathrm{s}}-\gamma_{\mathrm{gb}}\right)$ [18]. Based on this relationship, they proposed a mechanism for improving the 
fracture toughness. In atomic models, the deformation site for obtaining the plastic strain near the intergranular crack tip depends on the crack propagation direction. Specifically, the plastic strain should be determined at the GBs of right cracks and at the tips of left cracks. In right cracks, the dislocation source density of the GBs increases with increasing deviation angle $(\Delta \theta)$ from the stable $\Sigma 11 \mathrm{~GB}$. As is evident in Fig. 10, the $\gamma_{\mathrm{p}}$ caused by dislocation emission from GBs and the disclination shielding effect increases with $\Delta \theta$. Conversely, the $\gamma_{\mathrm{p}}$ induced by dislocation emission from a left crack tip decreases with increasing $\Delta \theta$ (see Fig. 11(a) and (b)). Since the GB energies adopted in this study increase as $\Delta \theta$ increases; $\left(2 \gamma_{\mathrm{s}}-\gamma_{\mathrm{gb}}\right)$ decreases with increasing $\Delta \theta$. This result implies that, when GBs act as dislocation sources, $\gamma_{\mathrm{p}}$ is not necessarily an increasing function of $\left(2 \gamma-\gamma_{\mathrm{gb}}\right)$. Therefore, the role of GBs as dislocation source cannot be ignored when designing nanostructured materials with high fracture toughness based on GB engineering [28].

Finally, we consider the size effect of the crack tip plasticity, and its relation to disclination shielding by dislocations emitted from GBs. The most important phenomenon is the stability of disclinations in crystalline materials. Although perfect disclinations are highly unlikely to persist in crystalline materials, partial disclinations accompanied by plane defects [31] have been observed in grains, GBs, and their junctions [45-47]. The extent of disinclination depends on the magnitude of $\omega ; \omega$ is closely related to the crystalline structure in perfect disclinations but is arbitrary in partial disclinations. All disclinations observed in this study were partial wedge disclinations. In Zhou et al.'s study of partial disclination in a nanowire, the magnitude of the stable Frank vector increased as the wire diameter decreased [48]; hence, if the GBs in polycrystalline materials can shield the mechanical field caused by disclinations, as those occurring on free surfaces, large- $\omega$ disinclinations can stably exist in UFG materials. The assumption suggests the size effect of the dislocation emission from the GB accompanied by disclination formation: smaller grain size facilitates the dislocation emission from GBs and increases the disclination shielding effect. These size effects can also explain the improved fracture toughness of UFGs at low temperatures [24, 25]. Therefore, elucidating the extent to which GBs shield the mechanical fields of lattice defects is very important and requires investigation in future analyses. 


\section{Conclusion}

In this study, we investigated the atomic-scale mechanism of intergranular crack tip plasticity in aluminum $<112>$ tilt GBs, focusing on GBs as effective dislocation sources. The investigations combined continuum analyses with atomic simulations. To quantitatively predict the first plastic deformation near the intergranular crack tip, we first modeled the dislocation emission from the GBs ahead of the intergranular crack tip and analytically derived the critical stress intensity factor. When dislocation emission from GBs was predicted as the first plastic phenomenon by the continuum analyses, the stress field near the crack was shielded by a wedge disclination caused by continuous dislocation emission from the GBs. Although dislocations were much more readily emitted from crack tips than from GBs in the continuum models, the GBs significantly contributed to dislocation emission in the atomic models. As the lattice defect evolved, a nanograin was nucleated with a disclination at the triple-junction ahead of the crack tip. This microstructure also weakened the mechanical field near the crack tip. We conclude that the intergranular fracture toughness is influenced by both the energy and structure of the GB and that GBs play an important role as dislocation sources.

\section{Acknowledgments}

The authors would like to thank K. Higashida and M. Yamaguchi for helpful discussions. This research was supported by the Ministry of Education, Culture, Sports, Science and Technology (MEXT) KAKENHI Grant Number 22102007 and the Japan Science and Technology Agency (JST) under Collaborative Research Based on Industrial Demand "Heterogeneous Structure Control: Towards Innovative Development of Metallic Structural Materials." 
Appendix A. Derivation of $f_{\mathrm{K}}, f_{\mathrm{d}^{2}}$, and $f_{\mathrm{dd}}$.

This appendix derives the direct crack-dislocation force $f_{\mathrm{K}}$, the self-image force $f_{\mathrm{d}^{2}}$, and the dislocation-dislocation interaction force $f_{\mathrm{dd}}$, from which we determine the critical SIF to dislocation emission from GBs.

The $f_{\mathrm{K}}$ can be simply calculated from the SIF $K_{\mathrm{I}}$, which is a function of the applied load $\sigma_{\mathrm{A}}$ and the crack length. The stress components due to $K_{\mathrm{I}}$ at dislocation $\zeta$ shown in Fig. 3(a) are given by

$$
\begin{aligned}
\sigma_{x} & =\frac{K_{\mathrm{I}}}{\sqrt{2 \pi r^{\prime}}} \cos \frac{\psi}{2}\left(1-\sin \frac{\psi}{2} \sin \frac{3 \psi}{2}\right)-\sigma_{\mathrm{A}}, \\
\sigma_{z} & =\frac{K_{\mathrm{I}}}{\sqrt{2 \pi r^{\prime}}} \cos \frac{\psi}{2}\left(1+\sin \frac{\psi}{2} \sin \frac{3 \psi}{2}\right), \\
\tau_{x z} & =\frac{\mathrm{K}_{\mathrm{I}}}{\sqrt{2 \pi r^{\prime}}} \sin \frac{\psi}{2} \cos \frac{\psi}{2} \cos \frac{3 \psi}{2} .
\end{aligned}
$$

The second term $\sigma_{\mathrm{A}}$ in Eq. (A.1) is usually neglected. However, because the crack length in our atomic model is very short, $\sigma_{\mathrm{A}}$ exerts a non-negligible effect on $\sigma_{x} . f_{\mathrm{K}}$ is evaluated as $b \tau_{r \theta} . b$ is the magnitude of the Burgers vector, and $\tau_{r \theta}$ is calculated by a coordinate transformation of Eqs. (A.1), (A.2), and (A.3).

Second, $f_{\mathrm{d}^{2}}$ is determined from the stress fields of image dislocations on the crack plane. As shown in Fig. 3, dislocation $\zeta$ elastically interacts with the crack. To distinguish the stress components $\sigma_{z}$ and $\tau_{z x}$ along the crack surface, image dislocation distributions are introduced along the crack surface [49]. The image dislocation distributions at distance $\beta$ from the crack tip, caused by dislocation $\zeta$, are given by

$$
\begin{aligned}
& F_{x}(\beta)=-\frac{b}{\pi R} \sqrt{\frac{r^{\prime}}{-\beta}}\left\{\cos \eta \cos \left(\phi-\frac{\psi}{2}\right)+\frac{1}{2} \sin \psi \sin \left(\phi-\eta+\frac{\psi}{2}\right)-\sin \phi \sin \left(2 \phi-\eta-\frac{\psi}{2}\right)\right\}, \\
& F_{z}(\beta)=-\frac{b}{\pi R} \sqrt{\frac{r^{\prime}}{-\beta}}\left\{2 \sin \eta \cos \left(\phi-\frac{\psi}{2}\right)+\cos \left(2 \phi-\frac{\psi}{2}\right) \sin (\phi-\eta)-\frac{1}{2} \sin \psi \cos \left(\phi-\eta+\frac{\psi}{2}\right)\right\} .
\end{aligned}
$$

Here, $F_{x}(\beta) \mathrm{d} \beta$ represenis the sum of the $x$-directional components of the Burgers vector of the image dislocations between $\beta$ and $\beta+\mathrm{d} \beta$. Substituting Eqs. (A.4) and (A.5) for Burgers vector 
in the stress field equations of a discrete dislocation [41] and integrating the functions over $\beta$ along the crack surface, the stress fields imposed by all image dislocations are obtained as follows.

$$
\begin{aligned}
& \sigma_{x}=\frac{\mu}{2 \pi(1-v)}\left[-\int_{-\infty}^{0} \frac{z\left\{3(x-\beta)^{2}+z^{2}\right\}}{\left\{(x-\beta)^{2}+z^{2}\right\}^{2}} F_{x}(\beta) \mathrm{d} \beta+\int_{-\infty}^{0} \frac{(x-\beta)\left\{(x-\beta)^{2}-z^{2}\right\}}{\left\{(x-\beta)^{2}+z^{2}\right\}^{2}} F_{z}(\beta) \mathrm{d} \beta\right], \\
& \sigma_{z}=\frac{\mu}{2 \pi(1-v)}\left[\int_{-\infty}^{0} \frac{z\left\{(x-\beta)^{2}-z^{2}\right\}}{\left\{(x-\beta)^{2}+z^{2}\right\}^{2}} F_{x}(\beta) \mathrm{d} \beta+\int_{-\infty}^{0} \frac{(x-\beta)\left\{(x-\beta)^{2}+3 z^{2}\right\}}{\left\{(x-\beta)^{2}+z^{2}\right\}^{2}} F_{z}(\beta) \mathrm{d} \beta\right], \\
& \tau_{x z}=\frac{\mu}{2 \pi(1-v)}\left[\int_{-\infty}^{0} \frac{(x-\beta)\left\{(x-\beta)^{2}-z^{2}\right\}}{\left\{(x-\beta)^{2}+z^{2}\right\}^{2}} F_{x}(\beta) \mathrm{d} \beta+\int_{-\infty}^{0} \frac{z\left\{(x-\beta)^{2}-z^{2}\right\}}{\left\{(x-\beta)^{2}+z^{2}\right\}^{2}} F_{z}(\beta) \mathrm{d} \beta\right] .
\end{aligned}
$$

Here, the interval of integration is set $-100 \mathrm{~nm}<\beta<0$, and the integration is done numerically in this study. Consequently, $f_{\mathrm{d}^{2}}$ can be calculated by the same manner of $f_{\mathrm{K}}$.

Finally, $f_{\mathrm{dd}^{\prime}}$ can be calculated by considering the direct and indirect interaction forces $f_{\mathrm{dd}^{\prime}, \mathrm{d}}$ and $f_{\mathrm{dd}, \text {, }}$ caused by the residual dislocation $\zeta^{\prime}$ after the dislocation $\zeta$ emission from the GB, as shown in Fig. 3. Dislocation $\zeta^{\prime}$ makes two types of stress fields to dislocation $\zeta$ : the direct and indirect stress fields. The force $f_{\mathrm{dd}^{\prime}, \mathrm{d}}$ caused by the direct stress filed can be easily evaluated because dislocations $\zeta$ and $\zeta^{\prime}$ are on the same atomic plane [41].

$$
f_{\mathrm{dd}^{\prime}, \mathrm{d}}=-\frac{\mu b^{2}}{2 \pi(1-v) r}
$$

On the other hand, the indirect force $f_{\mathrm{dd}^{\prime}, \mathrm{i}}$ can be calculated in the same manner as $f_{\mathrm{d}^{2}}$. The image dislocation distributions caused by dislocation $\zeta^{\prime}$ is obtained by setting $b=-b, R=\alpha-$ $\beta, r^{\prime}=\alpha$, and $\psi=\phi=0$ for Eqs. (A.4) and (A.5). The obtained distributions are substituted for Eqs. (A6), (A7), and (A8), and $f_{\mathrm{dd}^{\prime}, \mathrm{i}}$ can be evaluated.

\section{Appendix B. Correlation of stress intensity factor}

We correlate the SIF $K_{\mathrm{I}}$ to express the stress field near the crack tip in atomic models. Because we will compare the first plastic deformation near the intergranular crack tip observed in atomic simulations with that predicted by the continuum analyses proposed in sec. 2.3 , it 
will be a problem if the crack tip stress fields in Eqs. (A.1), (A.2), and (A.3) are quite different from that of atomic models. Figure B.1 shows the stress distributions $\sigma_{x}$ and $\sigma_{z}$ ahead of a crack tip along the crack plane with circles by an atomic model and with blue broken lines by Eqs. (A.1) and (A.2). As mentioned before, the crack tip position is shifted a distance of $\rho / 2$ behind the physical tip. A single crystalline model with the same crystal orientation of $\Sigma 175$ GB is employed and the dimensions of the model are the same as the $\Sigma 175$ GB model as shown in Table 1 . The analysis temperature is $1 \mathrm{~K}$. The geometry correction factor $F_{1}=1.439$ due to the free surface in the $x$-direction is considered [38]. In case of $\sigma_{x}$, both values show good agreement, but for $\sigma_{z}$, the elastic theory overestimates the field of the atomic model. Therefore, we introduce an additional correction factor $F_{2}$. Red lines in Fig. 4 shows the stress distributions of the elastic theory with $F_{2}=0.91$ and that values can express results of atomic models better than blue lines. Consequently, we adopt $F_{1} F_{2} K_{\mathrm{I}}$ for the SIF in Eqs. (A.1), (A.2), and (A.3).

\section{Appendix C. Critical state of dislocation nucleation}

To evaluate the critical SIF to the first plastic deformation near the crack tip $K^{\mathrm{MD}}$ in atomic simulations, the critical state of a dislocation nucleation is determined. Figure C.1 (a) shows the relationship between the applied stress $\sigma_{\mathrm{A}}$ and the distance $r_{\mathrm{d}}$ of the first leading partial dislocation from the right crack tip in $\Sigma 73 \mathrm{~GB}$ model. Here, $\alpha_{(4)}=1.4 \mathrm{~nm}$. Atomic configurations near the crack tip are shown in Fig. C.1 (b-d). When $\sigma_{\mathrm{A}}$ becomes larger than 1 $\mathrm{GPa}$, the leading partial dislocation begins to nucleate toward the left lower direction from the crack tip and $r_{\mathrm{d}}$ takes less than $1 \mathrm{~nm}$, as shown in Fig. C.1 (b). Then, $r_{\mathrm{d}}$ increases dramatically when $\sigma_{\mathrm{A}}$ reaches at $1.3 \mathrm{GPa}$; hence, the critical state can be regarded as the atomic configuration in Fig. C.1 (c). Consequently, in this study, we determine the critical state of dislocation nucleation from a crack tip or GBs as the situation when $r_{\mathrm{d}}$ reaches approximately $1.4 \mathrm{~nm}$ ( $5 \mathrm{hcp}$ atoms line up on the slip plane.). It should be noted that the center position $r_{\mathrm{d}} / 2$ of the emitting dislocation in the critical state agrees well to the cut off value $r_{0}=0.75 \mathrm{~nm}$ obtained by Eq. (6). Because the increasing rate of $r_{\mathrm{d}}$ during the dislocation emission from the crack tip is much faster than that of the applied stress as shown in Fig. C1(a), the choice of $r_{\mathrm{d}}$ to determine the critical state has negligible impact on the critical SIF. 


\section{References}

[1] H. Gleiter, Nanostructured materials: basic concepts and microstructure, Acta Mater. 48(2000) 1-29.

[2] R. Valiev, Nanostructuring of metals by severe plastic deformation for advanced properties, Nature Mater. 3(2004) 511-516.

[3] Y. Wang, M. Chen, F. Zhou, E. Ma, High tensile ductility in a nanostructured metal, Nature 419(2002) 912-915.

[4] L. Lu, Y. Shen, X. Chen, L. Qian, K. Lu, Ultrahigh strength and high electrical conductivity in copper, Science 304(2004) 422-426.

[5] S.J. Noronha, D. Farkas, Effect of dislocation blocking on fracture behavior of Al and $\alpha-F e:$ a multiscale study, Mater. Sci. Eng. A 365(2004) 156-165.

[6] X. Zeng, A. Hartmaier, Modeling size effects on fracture toughness by dislocation dynamics, Acta Mater. 58(2010) 301-310.

[7] I.A. Ovid'ko, A.G. Sheinerman, Ductile vs. brittle behavior of pre-cracked nanocrystalline and ultrafine-grained materials, Acta Mater. 58(2010) 5286-5294.

[8] T.J. Anderson, Fracture Mechanics, Fundamentals and Applications CRC Press, Boca Raton, FL 1989.

[9] E. Orowan, Fracture and strength of solids, Rep. Prog. Phys. 12(1948) 185-232.

[10] G.R. Irwin, Fracture dynamics, in: F. Jonassen, W.P. Roop, R.T. Bayless (Eds.), Fracturing of Metals, Am. Soc. Metals, Cleveland 1948, pp.147-166.

[11] A.A. Griffith, The phenomena of rupture and flow in solids, Philos. Trans. R. Soc. London A, 221(1921) 163-198.

[12] J.R. Rice, R. Thomson, Ductile versus brittle behaviour of crystals, Philos. Mag. 29(1974) 73-97.

[13] A. Sutton, R. Balluffi, Interfaces in Crystalline Materials, Oxford Science, New York, 1995.

[14] D. Wolf, Correlation between structure, energy, and ideal cleavage fracture for symmetrical grain boundaries in fcc metals, J. Mater. Res. 5(1990) 1708-1730.

[15] J.W. Hutchinson, in: B.L. Karihaloo, Y.-W. Mai, M.I. Ripley, R.O. Ritchie (Eds.), Linking scales in fracture mechanics, Advances in Fracture Research, Pergamon Press, 
New York, 1997, pp. 1-14.

[16] M. Guttmann, D. McLean, in W.C. Johnson, J.M. Blakely (Eds.), Grain boundary segregation in multicomponent systems, Interfacial Segregation, Am. Soc. Metals, Metals Park, OH, 1979, pp. 261-347.

[17] S. Tsurekawa, T. Tanaka, H. Yoshinaga, Grain boundary structure, energy and strength in molybdenum, Mater. Sci. Eng. A 176(1994) 341-348.

[18] M.L. Jokl, V. Vitek, C.J. McMahon, Jr., A microscopic theory of brittle fracture in deformable solids: A relation between ideal work to fracture and plastic work, Acta Metall. 28(1980) 1479-1488.

[19] M. Yamaguchi, J. Kamada, Multiscale thermodynamic analysis on fracture toughness loss induced by solute segregation in steel, Philo. Mag. 94(2014) 2131-2149.

[20] K.S. Kumar, H. Van Swygenhoven, S. Suresh, Mechanical behavior of nanocrystalline metals and alloys, Acta Mater. 51(2003) 5743-5774.

[21] S. Cheng, J.A. Spencer, W.W. Milligan, Strength and tension/compression asymmetry in nanostructured and ultrafine-grain metals, Acta Mater. 51(2003) 4505-4518.

[22] M. Kato, Thermally activated dislocation depinning at a grain boundary in nanocrystalline and ultrafine-grained materials, Mater. Sci. Eng. A 516(2009) 276-282.

[23] Y. Mishin, M. Asta, J. Li, Atomistic modeling of interfaces and their impact on microstructure and properties, Acta Mater. 58(2010) 1117-1151.

[24] N. Tsuji, S. Okuno, Y. Koizumi, Y. Minamino, Toughness of ultrafine grained ferritic steels fabricated by ARB and annealing process, Mater. Trans. 45(2004) 2272-2281.

[25] M. Tanaka, K. Higashida, T. Shimokawa, T. Morikawa, Brittle-ductile transition in low carbon steel deformed by the accumulative roll bonding process, Mater. Trans. 50(2009) 56-63.

[26] T. Shimokawa, M. Tanaka, K. Kinoshita, K. Higashida, Roles of grain boundaries in improving fracture toughness of ultrafine-grained metals, Phys. Rev. B, 83(2011) 214113(1-13).

[27] D.E. Spearot, M.A. Tschopp, K.I. Jacob, D.L. McDowell, Tensile strength of $<100>$ and $<110>$ tilt bicrystal copper interfaces, Acta Mater. 55(2007) 705-714.

[28] M.A. Tschopp, D.L. McDowell, Dislocation nucleation in $\Sigma 3$ asymmetric tilt grain boundaries, Int. J. Plast. 24(2008) 191-217. 
[29] T. Shimokawa, Asymmetric ability of grain boundaries to generate dislocations under tensile or compressive loadings, Phys. Rev. B 82(2010) 174122(1-13).

[30] T. Watanabe, S. Tsurekawa, The control of brittleness and development of desirable mechanical properties in polycrystalline systems by grain boundary engineering, Acta Mater. 47(1999) 4171-4185.

[31] A.E. Romanov, V.I. Vladimirov, Disclinations in crystalline solids, in: F.R.N. Nabarro (Ed.), Dislocations in Solids, Vol.9, North-Holland, Amsterdam, 1992, pp. 191-402.

[32] Y. Mishin, D. Farkas, M.J. Mehl, D.A. Papaconstantopoulos, Interatomic potentials for monoatomic metals from experimental data and $a b$ initio calculations, Phys. Rev. B 59(1999) 3393-3407.

[33] V. Yamakov, E. Saether, D.R. Phillips, E.H. Glaessgen, Molecular-dynamics simulation-based cohesive zone representation of intergranular fracture processes in aluminum, J. Mech. Phys. Solid. 54(2006) 1899-1928.

[34] Y. Cheng, Z.-H. Jin, Y.W. Zhang, H. Gao, On intrinsic brittleness and ductility of intergranular fracture along symmetrical tilt grain boundaries in copper, Acta Mater. 58(2010) 2293-2299.

[35] J.J. Möller, E. Bitzek, Fracture toughness and bond trapping of grain boundary cracks, Acta Mater. 73(2014) 1-11.

[36] M.P. Allen, D.J. Tildesley, Computer Simulation of Liquids, Oxford University Press, New York, 1987.

[37] J. Dana Honeycutt, H.C. Andersen, Molecular dynamics study of melting and freezing of small Lennard-Jones clusters, J. Phys. Chem. 91(1987) 4950-4963.

[38] M. Janssen, J. Zuidema, R. Wanhill, Fracture Mechanics (2 ${ }^{\text {nd }}$ ed.) VSSD, Delft, 2006.

[39] J.R. Rice, Dislocation nucleation from a crack tip: An analysis based on the Peierls concept, J. Mech. Phys. Solids. 40(1992) 239-271.

[40] W. Voigt, Lehrbuch der Kristallphysik, Teubner, Leipzig, 1928.

[41] J.P. Hirth, J. Lothe, Theory of Dislocations, New York, McGraw-Hill, 1968.

[42] N.F. Morozov, I.A. Ovid'ko, A.G. Sheinerman, E.C. Aifantis, Special rotational deformation as a toughening mechanism in nanocrystalline solids, J. Mech. Phys. Solids 58(2010) 1088-1099.

[43] I.A. Ovid'ko, N.V. Skiba, A.K. Mukherjee, Nucleation of nanograins near cracks in 
nanocrystalline materials, Scripta Mater. 62(2010) 387-390.

[44] K. Zhou, A.A. Nazarov, M.S. Wu, Atomistic simulations of the tensile strength of a disclinated bicrystalline nanofilm, Philo. Mag. 88(2008) 3181-3191.

[45] M. Murayama, J.M. Howe, H. Hidaka, S. Takaki, Atomic-Level Observation of Disclination Dipoles in Mechanically Milled, Nanocrystalline Fe, Science 295(2002) 2433-2435.

[46] A.E. Romanov, A.L. Kolesnikova, Application of disclination concept to solid structures, Prog. Mater. Sci. 54(2009) 740-769.

[47] B. Beausir, C. Fressengeas, Disclination densities from EBSD orientation mapping, Int. J. Solids Struct. 50(2013)137-146.

[48] K. Zhou, A.A. Nazarov, M.S. Wu, Continuum and atomistic studies of a disclinated crack in a bicrystalline nanowire, Phys. Rev. B 73(2006) 045410(1-11).

[49] V. Lakshmanan, J.C.M. Li, Edge dislocations emitted along inclined planes from a mode I crack, Mater. Sci. Eng. A 104(1988) 95-104. 


\section{Captions of figures}

Fig. 1 Atomic structures of $<112>$ tilt grain boundaries. Each grain boundary structure is represented by two types of structural units (distinguished by yellow and pink). The pink structural unit contains a grain boundary dislocation relative to the reference structure (the $\Sigma 11$ boundary). $h$ denotes the spacing of the grain boundary dislocations.

Fig. 2 Schematic of the analysis model in the atomic simulations. (a) The analysis model comprises two grains, I and II (referred to as Grains 1 and 2 in main text), and an intergranular crack of length $L_{\mathrm{c}}$. A periodic boundary condition is applied in the $y$-direction. (b)-(e) Details of atomic configurations of left and right intergranular crack tips, varying the distance $\alpha$ between the crack tip and the pink structural unit.

Fig. 3 (a) Schematic of the analysis model for determining the stress intensity factor for dislocation emission from the grain boundary ahead of the intergranular crack tip. (b) Change of local grain boundary structure in GB0 caused by a dislocation $\zeta$ emission from GB1, composed of grain boundary dislocations $\kappa . \boldsymbol{b}^{\kappa}=\boldsymbol{b}^{\zeta}+\boldsymbol{b}^{\zeta^{\prime \prime}}$.

Fig. 4 Stress intensity factor for dislocation emission from the $C$ structural unit in various tilt grain boundaries ahead of the intergranular crack tip. Broken and solid lines are the results of our developed theory and the modified theory (1.15 $f_{\text {gbe }}$ in Eq. (12)), respectively. 
Fig. 5 Atomic configurations around the left crack tip of various GBs: (a) and (b) $\Sigma 77$ with $\alpha_{(2)}=0.9 \mathrm{~nm}$, (c) and (d) $\Sigma 73$ with $\alpha_{(4)}=1.4 \mathrm{~nm}$, and (e) and (f) $\Sigma 175$ with $\alpha_{(11)}=3.4 \mathrm{~nm}$. (b), (c), and (e) represent the critical state for dislocation emission from the grain boundaries. (a) $K$ $=0.200 \mathrm{MPam}^{1 / 2}$. (b) A dislocation emission occurs at the second $C$ structural unit from the crack tip. $K_{\text {disl,gb }}^{\mathrm{MD}}=0.442 \mathrm{MPam}^{1 / 2}$. Structural changes ahead of the crack tip occur. (c) $K_{\text {disl,gb }}^{\mathrm{MD}}=0.344 \mathrm{MPam}^{1 / 2}$. (d) The leading partial is strongly trapped by the left crack tip. $K=$ $0.400 \mathrm{MPam}^{1 / 2}$. (e) $K_{\mathrm{disl}, \mathrm{gb}}^{\mathrm{MD}}=0.413 \mathrm{MPam}^{1 / 2}$. (f) The dislocation is emitted from the $C$ structural unit. The step in the broken circle is caused by the Burgers vector of the grain boundary dislocation $\zeta "$ being parallel to the plane. $K_{\mathrm{disl}, \mathrm{gb}}^{\mathrm{MD}}=0.484 \mathrm{MPam}^{1 / 2}$.

Fig. 6 Analytical and modeled tress intensity factors for plastic phenomena around the right intergranular crack tip. Solid (open) and broken (solid) lines (symbols) represent the estimated (observed) stress intensity factors for dislocation emission from the crack tip and from the grain boundaries ahead of the crack tip (1.15 $f_{\text {gbe }}$ in Eq. (5)), respectively.

Fig. $7 \sigma_{z}$ stress distributions around the right crack tip of the $\Sigma 175$ GB with (a) $\alpha_{(3)}=1.1 \mathrm{~nm}$ and (b) $\alpha_{(10)}=3.1 \mathrm{~nm}$. (d) plots the $\alpha$ dependence of the crack tip local stress, $\sigma_{z}$,tip under no applied loading. The inset (c) of panel (a) shows the local region $S$ of $\sigma_{z}$,tip .

Fig. $8(a-c)$ Shift of stress concentration ahead of the left intergranular crack tip of the $\Sigma 15$ GB with $\alpha_{(2)}=0.9 \mathrm{~nm}$, caused by continuous dislocation emissions from the grain boundary. (d) Grain boundary transformation from $\Sigma 15$ to $\Sigma 11$ caused by dislocation emission from the grain boundary ahead of the left crack. Notice that when lattice dislocations are emitted from $C$-SUs into the upper and lower grains alternately, residual dislocations $\zeta$ " can be annihilated by sliding on the GB plane; hence, the $\Sigma 11$ boundary plane becomes almost completely flat. 
Fig. 9 Schematic of lattice defect evolutions ahead of the (a) left and (b) right intergranular crack tips. (a) A wedge disclination dipole with $\omega_{1}$. (b) A nanograin with a wedge disclination with $\omega_{3}$ at the triple junction among the original GB, $\Sigma 11$, and LAGB.

Fig. 10 Stress distributions around left crack tips with negative disclination and the same grain boundary locations of $\omega_{1}$. The wedge disclination forms at the border of $\Sigma 11$ and a high angle grain boundary. (a) $\Sigma 175$ with $\alpha_{(4)}=1.4 \mathrm{~nm}$ when $K_{\mathrm{I}}=0.55 \mathrm{MPam}^{1 / 2}$. (b) $\Sigma 73$ with $\alpha_{(3)}=$ $1.2 \mathrm{~nm}$ when $K_{\mathrm{I}}=0.68 \mathrm{MPam}^{1 / 2}$. (c) $\Sigma 77$ with $\alpha_{(2)}=0.9 \mathrm{~nm}$ when $K_{\mathrm{I}}=0.70 \mathrm{MPam}^{1 / 2}$. (c) $\Sigma 15$ with $\alpha_{(2)}=0.9 \mathrm{~nm}$ when $K_{\mathrm{I}}=0.81 \mathrm{MPam}^{1 / 2} \cdot \sigma_{z}$ around the disclination increases with increasing $\omega_{1}$.

Fig. 11 Nanograin formation near the right crack tip caused by dislocation emission from the crack tip and grain boundaries. (a) $\Sigma 77$ with $\alpha_{(4)}=1.5 \mathrm{~nm}$ at $\varepsilon_{z}=0.039$. (b) $\Sigma 73$ with $\alpha_{(7)}=2.3$ nm at $\varepsilon_{z}=0.042 .7$ th and 9 th dislocations emitted from each crack tip are trapped by low angle grain boundaries. Most residual dislocations $\zeta "$ making the step of GB plane moved toward the crack tip and disappeared; hence, the $\Sigma 11$ boundary plane becomes almost completely flat. Stress distributions around the nanograin ahead of the right crack tip in various GBs: (d) $\Sigma 77$ with $\alpha_{(4)}=1.5 \mathrm{~nm}$ at $\varepsilon_{z}=0.039$, (e) $\Sigma 73$ with $\alpha_{(7)}=2.3 \mathrm{~nm}$ at $\varepsilon_{z}=0.042$ and (f) $\Sigma 175$ with $\alpha_{(10)}=3.1 \mathrm{~nm}$ at $\varepsilon_{z}=0.042$. The grain boundaries split and migrate to form a negative wedge disclination with $\omega_{3}$ at the triple junction.

Fig. 12 Intergranular crack tip opening of $\Sigma 15$ boundary with $\alpha_{(3)}=1.2 \mathrm{~nm}$, induced by dislocation emissions from the right crack tip region. (a) $\varepsilon_{z}=0$, (b) $\varepsilon_{z}=0.025$, (c) $\varepsilon_{z}=0.030$, and (d) $\varepsilon_{z}=0.041$. Solid orange lines represent the original crack surface.

Fig. B.1 Comparison of stress distributions ahead of the crack tip (along the plane of the 28 
crack) computed analytically and by the atomic simulations. (a) $\sigma_{x}$ and (b) $\sigma_{z}$. The origin is shifted over a distance of $\rho / 2$ behind the crack tip of finite radius $\rho . F_{2}$ is a correction factor introduced to fit the results of the atomic simulations.

Fig. C.1 Position of the first leading partial emitted from the right intergranular crack tip of the $\Sigma 73$ boundary with $\alpha_{(4)}=1.4 \mathrm{~nm}$ under tensile loading. (c) shows the critical state, in which the critical width of the stacking fault is approximately $0.6 \mathrm{~nm}$. The gray, blue, and red colored atoms indicate nearest neighbor atomic configurations of the fcc structure, hcp structure, and other defects (defined using CNA), respectively. 
Table 1. Geometrical and mechanical properties of $<112>$ tilt grain boundaries. Structures are expressed as combinations of $B$ and $C$ structural units. $h$ represents the spacing of grain boundary dislocations. $\gamma_{\mathrm{gb}}$ and $\gamma_{\mathrm{s}}$ represent the grain boundary energy and surface energy, respectively. $L_{\mathrm{x}}$ is the length in the $x$-direction in the analysis models, and $F_{1}$ is the correction factor for the stress intensity factor. $\theta$ is the angle between the crack plane and the active slip plane in our models. $K_{\text {dis,ck }}^{\mathrm{Th}}$ and $K_{\text {cleav }}^{\mathrm{Th}}$ are the theoretical critical stress intensity factors for dislocation emission from the crack tip and cleavage fracture, respectively.

\begin{tabular}{|c|c|c|c|c|c|c|c|c|c|c|}
\hline GB & \multicolumn{2}{|c|}{$\Sigma 11$} & \multicolumn{2}{|c|}{$\Sigma 175$} & \multicolumn{2}{|c|}{$\Sigma 73$} & \multicolumn{2}{|c|}{$\Sigma 77$} & \multicolumn{2}{|c|}{$\Sigma 15$} \\
\hline$\theta_{\mathrm{GB}}\left[^{\circ}\right]$ & \multicolumn{2}{|c|}{63.0} & \multicolumn{2}{|c|}{67.5} & \multicolumn{2}{|c|}{70.0} & \multicolumn{2}{|c|}{72.6} & \multicolumn{2}{|c|}{78.5} \\
\hline structure & \multicolumn{2}{|c|}{$|B|$} & \multicolumn{2}{|c|}{$|(10 B) C|$} & \multicolumn{2}{|c|}{$|(6 B) C|$} & \multicolumn{2}{|c|}{$|(4 B) C|$} & \multicolumn{2}{|c|}{$|B B C|$} \\
\hline$h[\mathrm{~nm}]$ & \multicolumn{2}{|c|}{-} & \multicolumn{2}{|c|}{3.093} & \multicolumn{2}{|c|}{1.997} & \multicolumn{2}{|c|}{1.451} & \multicolumn{2}{|c|}{0.906} \\
\hline$\gamma_{\mathrm{gb}}\left[\mathrm{J} / \mathrm{m}^{2}\right]$ & \multicolumn{2}{|c|}{0.151} & \multicolumn{2}{|c|}{0.309} & \multicolumn{2}{|c|}{0.351} & \multicolumn{2}{|c|}{0.388} & \multicolumn{2}{|c|}{0.435} \\
\hline$\gamma_{\mathrm{s}}\left[\mathrm{J} / \mathrm{m}^{2}\right]$ & \multicolumn{2}{|c|}{1.013} & \multicolumn{2}{|c|}{1.023} & \multicolumn{2}{|c|}{1.027} & \multicolumn{2}{|c|}{1.032} & \multicolumn{2}{|c|}{1.040} \\
\hline$L_{x}[\mathrm{~nm}]$ & \multicolumn{2}{|c|}{80.06} & \multicolumn{2}{|c|}{88.26} & \multicolumn{2}{|c|}{80.96} & \multicolumn{2}{|c|}{82.35} & \multicolumn{2}{|c|}{80.78} \\
\hline$F_{1}$ & \multicolumn{2}{|c|}{1.503} & \multicolumn{2}{|c|}{1.439} & \multicolumn{2}{|c|}{1.495} & 1.4 & & 1.4 & 96 \\
\hline crack & left & right & left & right & left & right & left & right & left & right \\
\hline$\theta\left[^{\circ}\right]$ & 121.48 & 58.52 & 123.74 & 56.26 & 124.98 & 55.02 & 126.31 & 53.69 & 129.24 & 50.76 \\
\hline$K_{\mathrm{dis}, \mathrm{ck}}^{\mathrm{Th}}[\mathrm{MP} \sqrt{\mathrm{m}}]$ & 0.680 & 0.381 & 0.723 & 0.387 & 0.750 & 0.390 & 0.780 & 0.394 & 0.854 & 0.405 \\
\hline$K_{\text {cleav }}^{\mathrm{Th}}[\mathrm{MP} \sqrt{\mathrm{m}}]$ & 0.4 & & 0.3 & & 0.38 & & 0.3 & & 0.3 & 79 \\
\hline
\end{tabular}

Table 2. Frank vectors $\omega$ of wedge disclinations generated by dislocation emission from tilt grain boundaries. $\omega_{1}=\theta_{\mathrm{H}}-\theta_{\Sigma 11} \cdot \omega_{2}=\theta_{\mathrm{L}} \cdot \omega_{3}=\omega_{1}-\omega_{2}$.

\begin{tabular}{c|cccc}
\hline $\mathrm{GB}$ & $\Sigma 175$ & $\Sigma 73$ & $\Sigma 77$ & $\Sigma 15$ \\
\hline$\omega_{1}\left[^{\circ}\right]$ & 4.5 & 7.0 & 9.6 & 15.5 \\
$\omega_{2}\left[^{\circ}\right]$ & 6.4 & 10.0 & 14.1 & 23.6 \\
$\omega_{3}\left[^{\circ}\right]$ & -1.9 & -3.0 & -4.5 & -8.1 \\
\hline
\end{tabular}


Figure 2
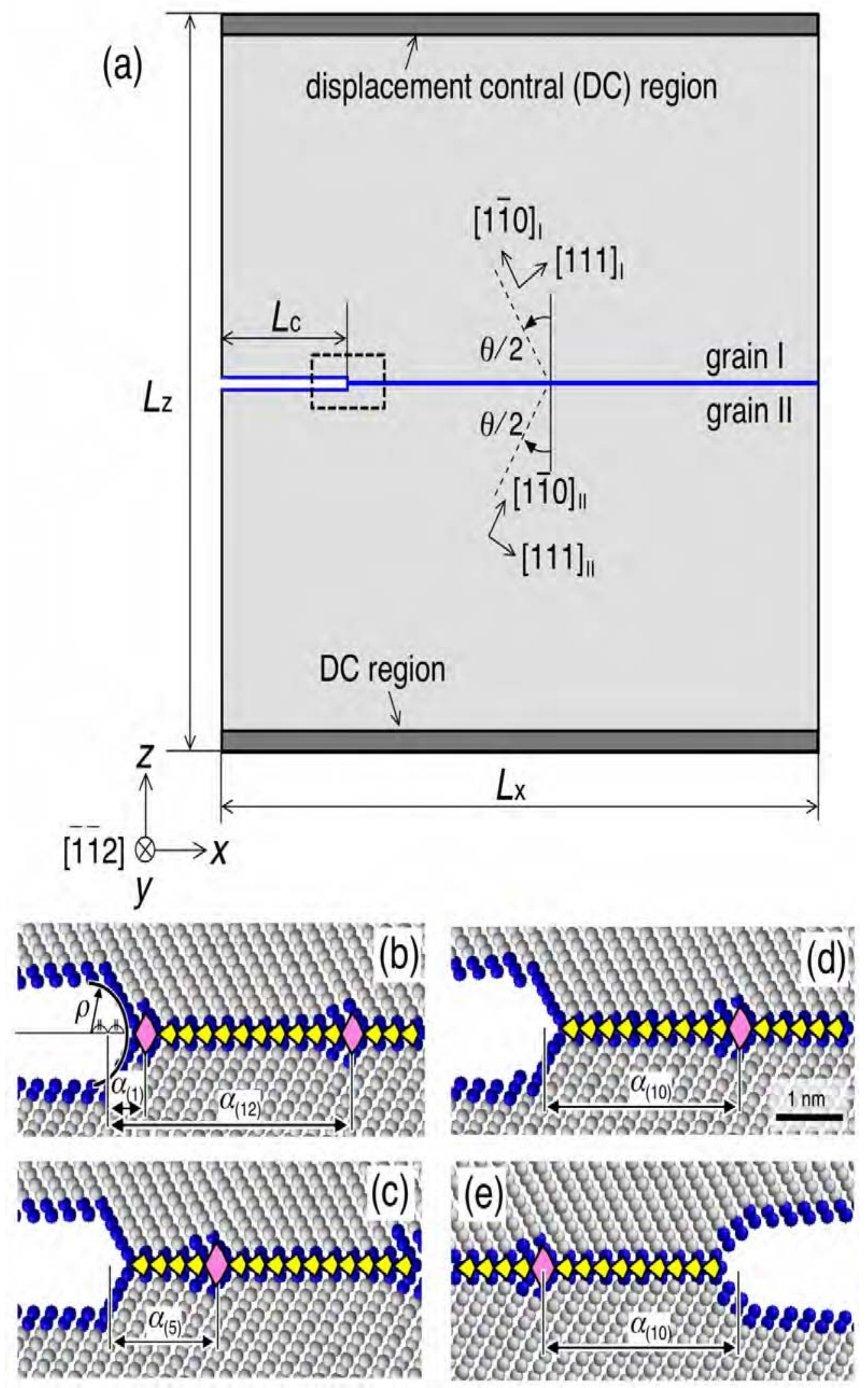
Figure 3

(a)

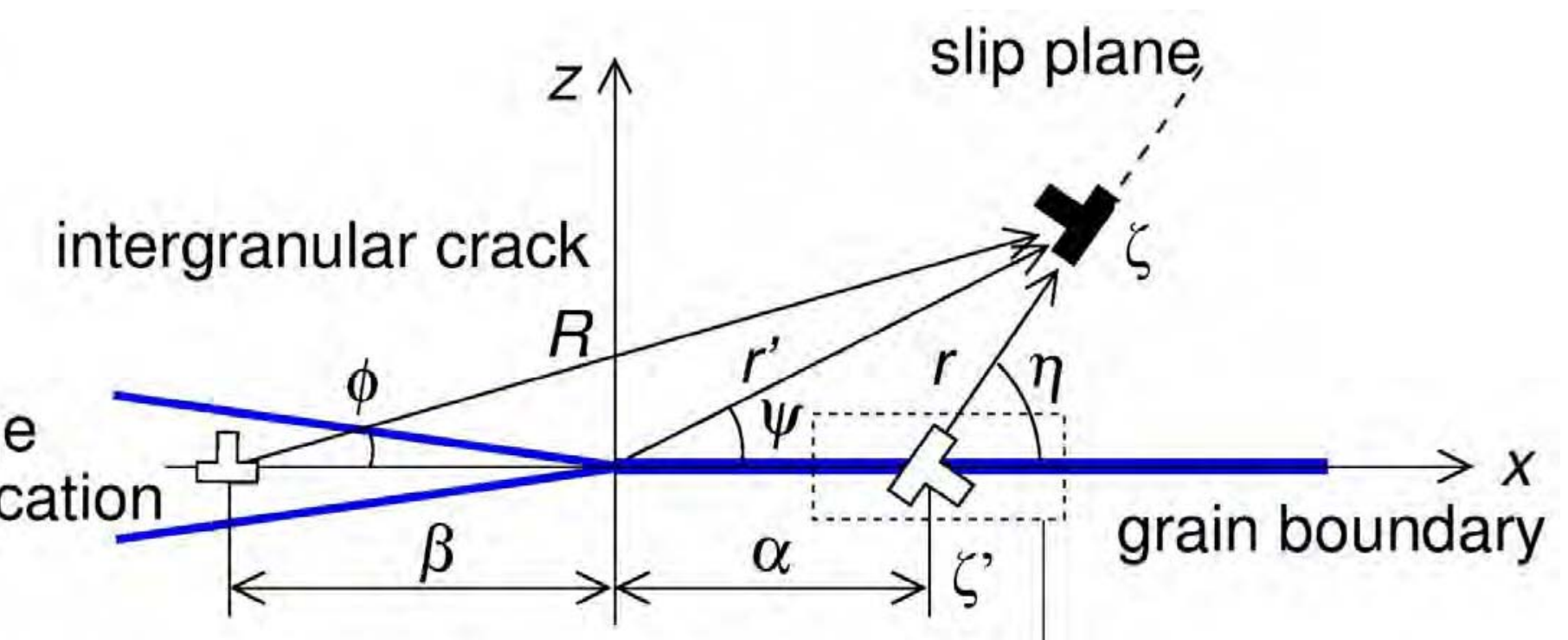

image dislocation
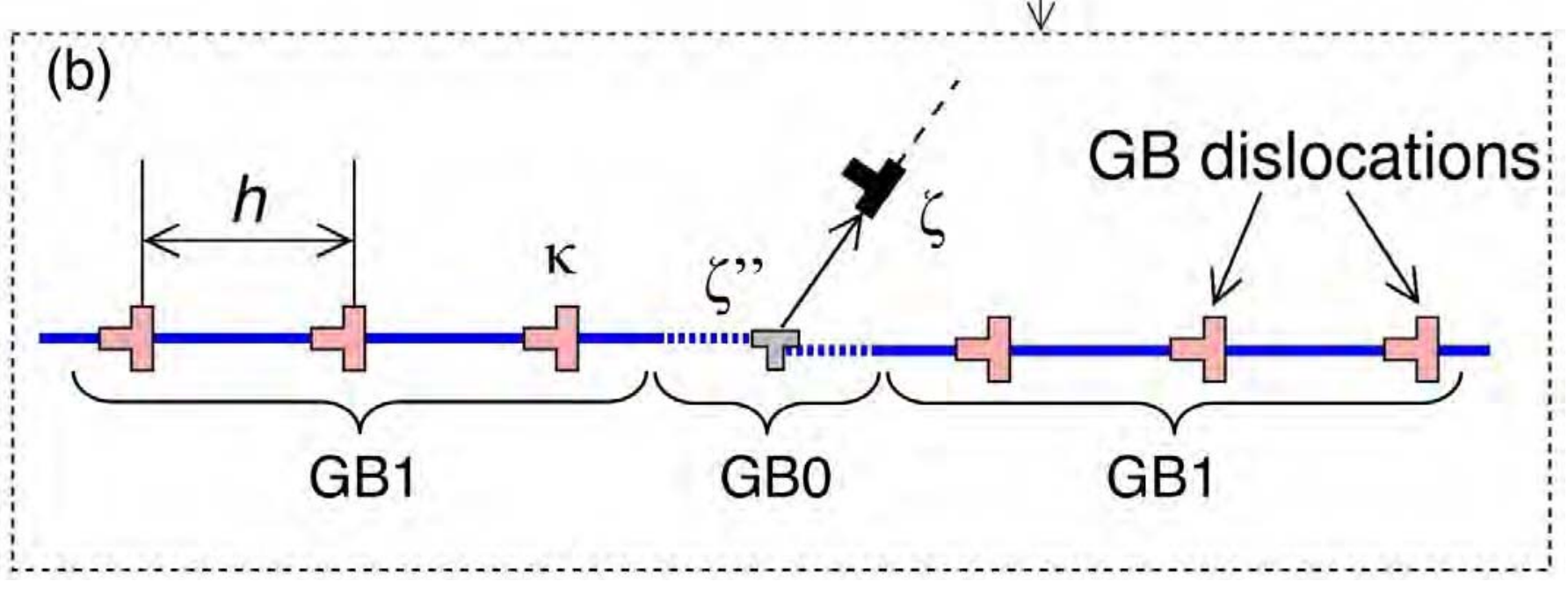

$\downarrow$ 
Figure 4

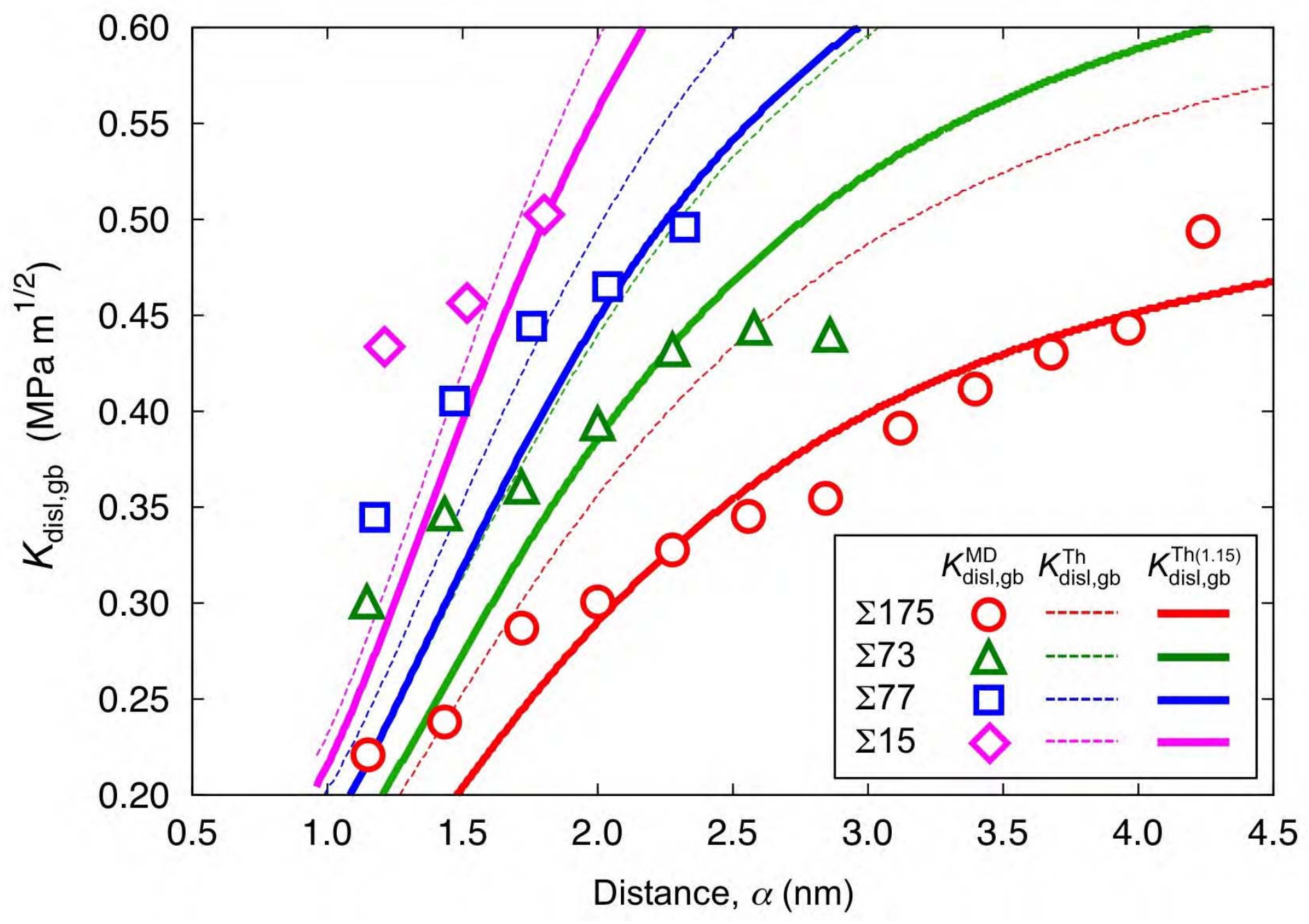


Figure 5

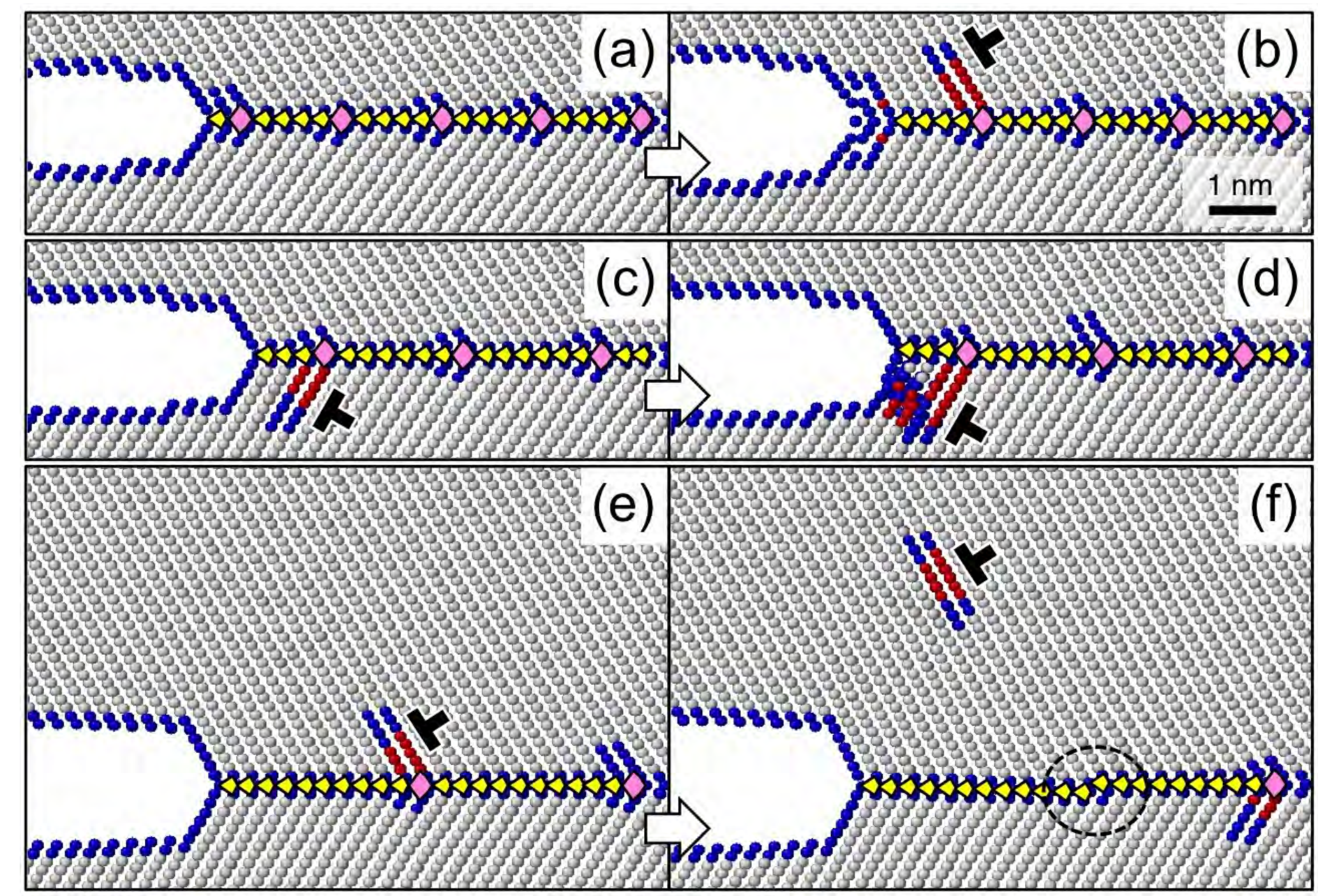


Figure 6

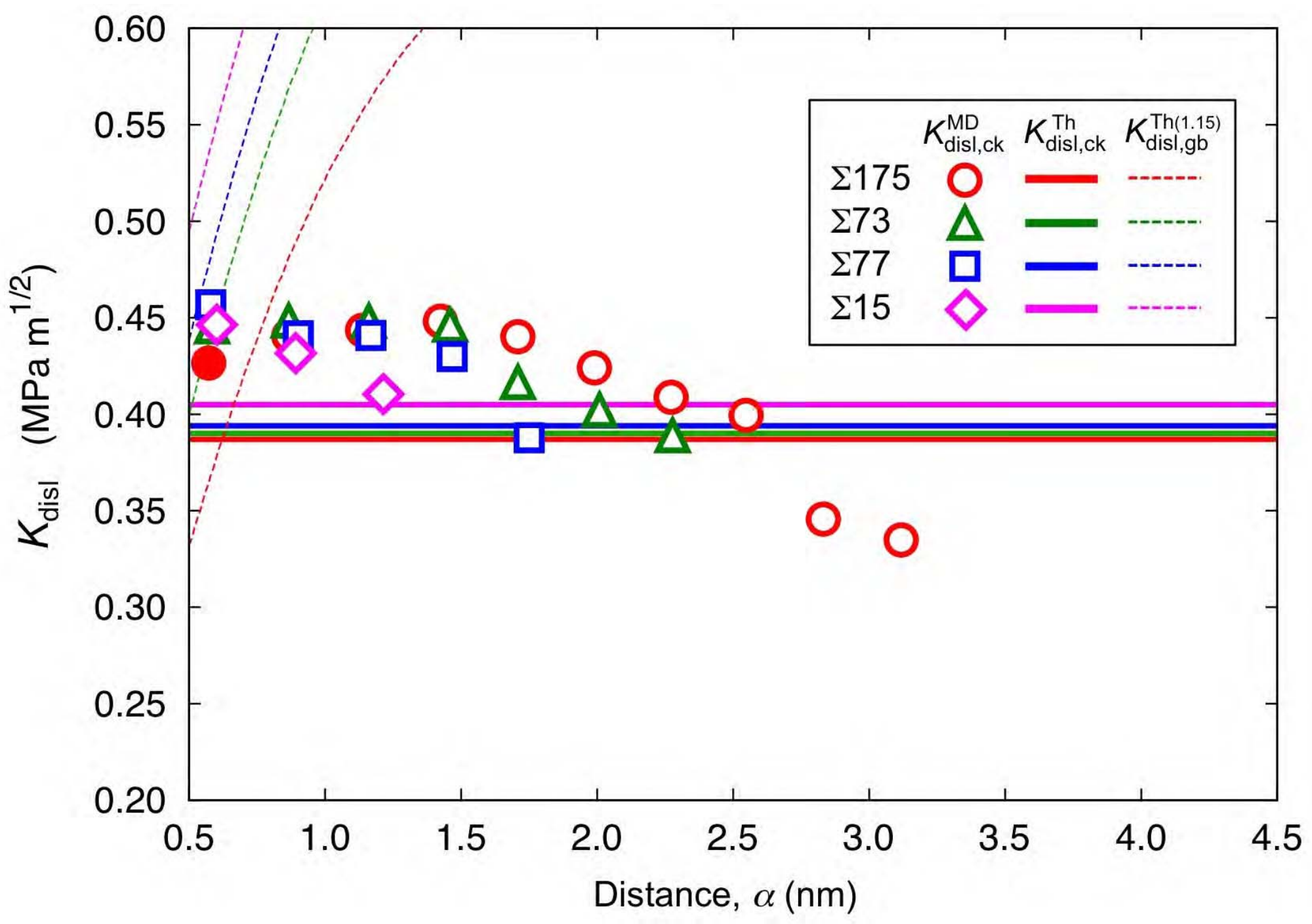


Figure 7
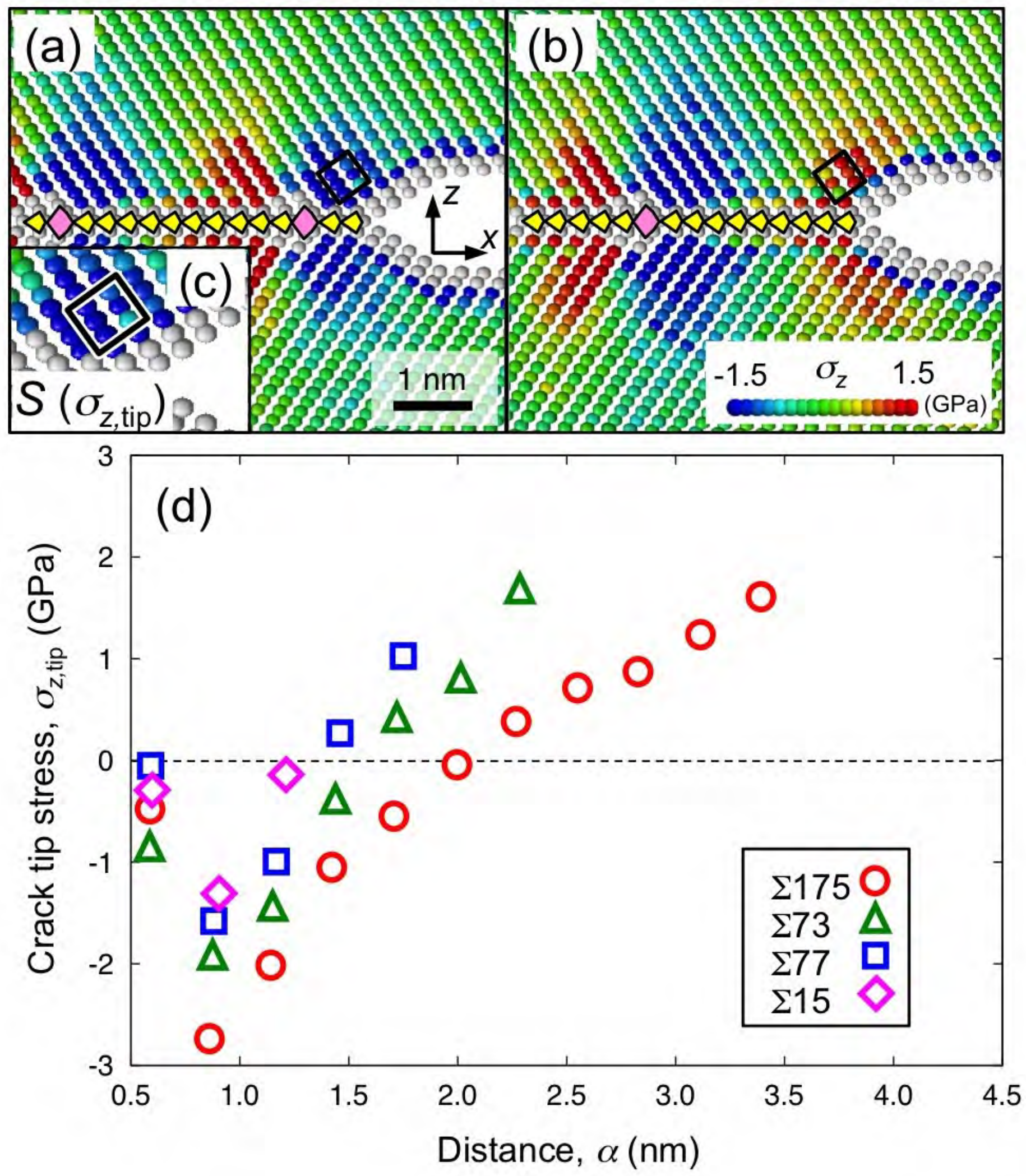
Figure 8
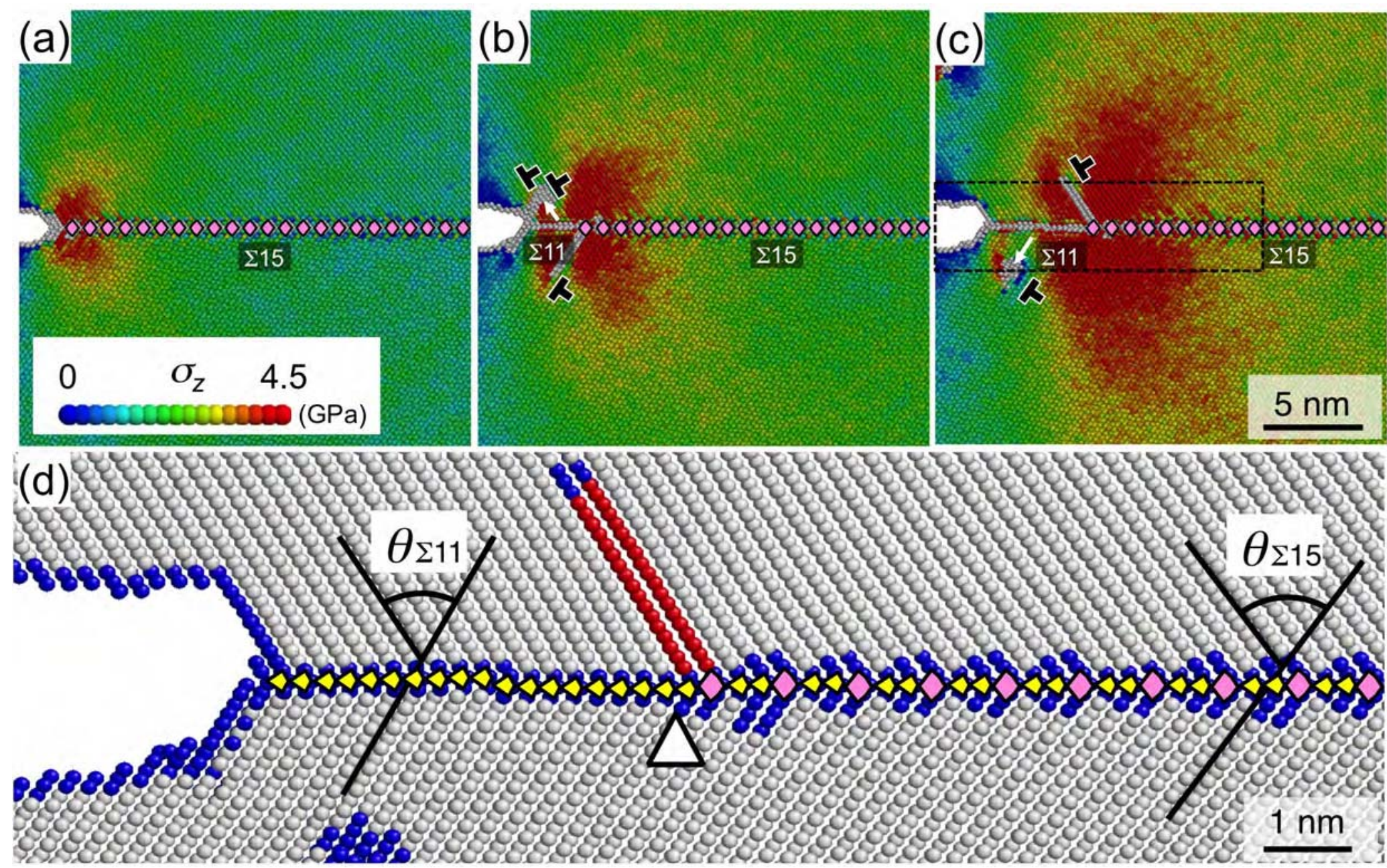
Figure 9
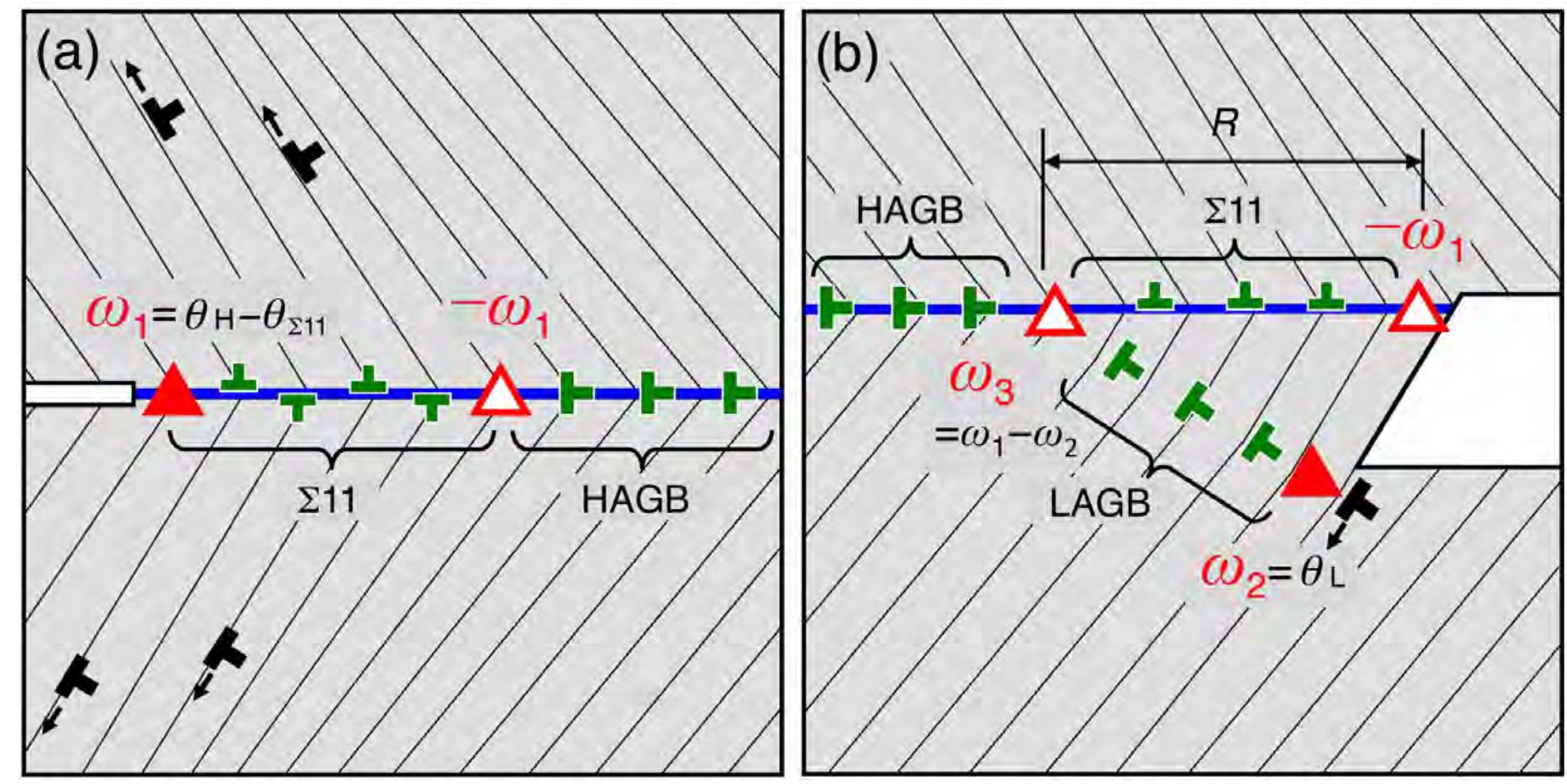
Figure 10
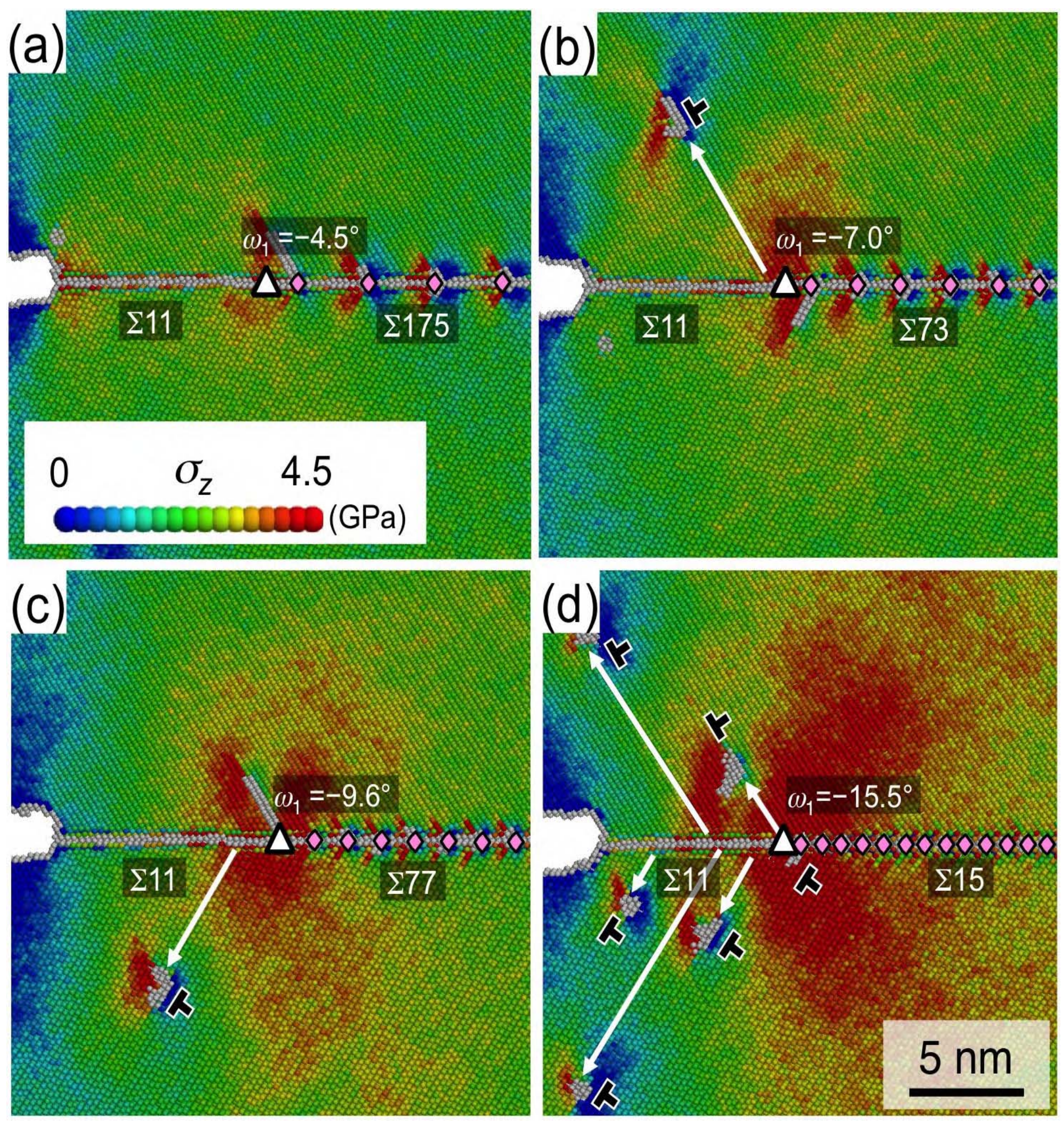


\section{Figure 11}

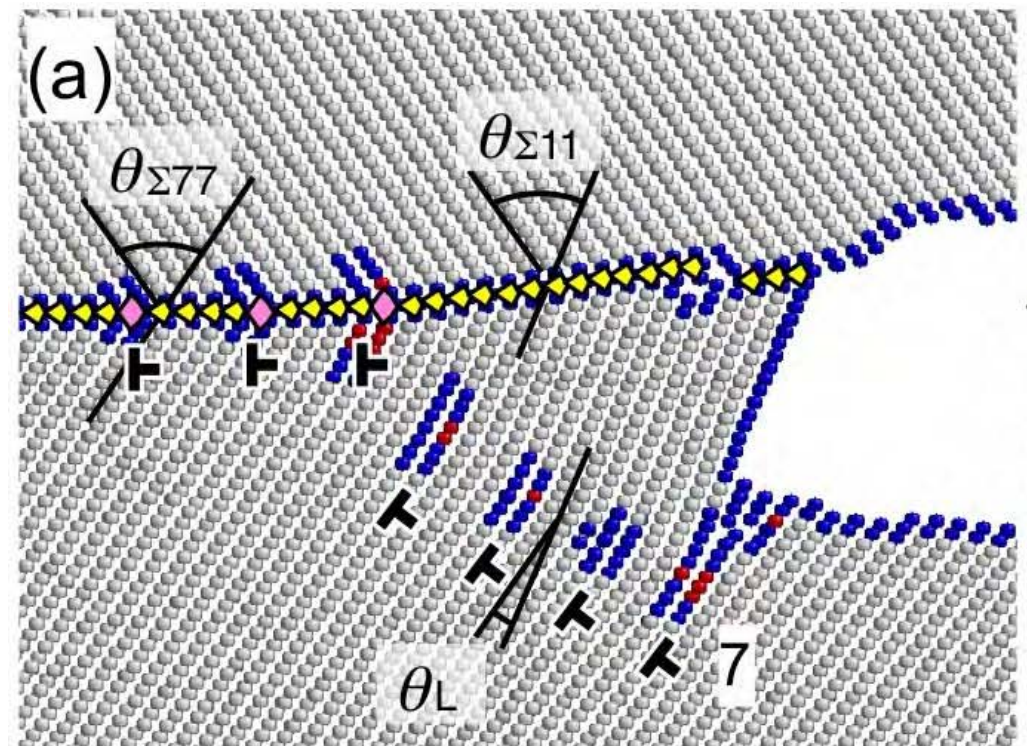

(b)
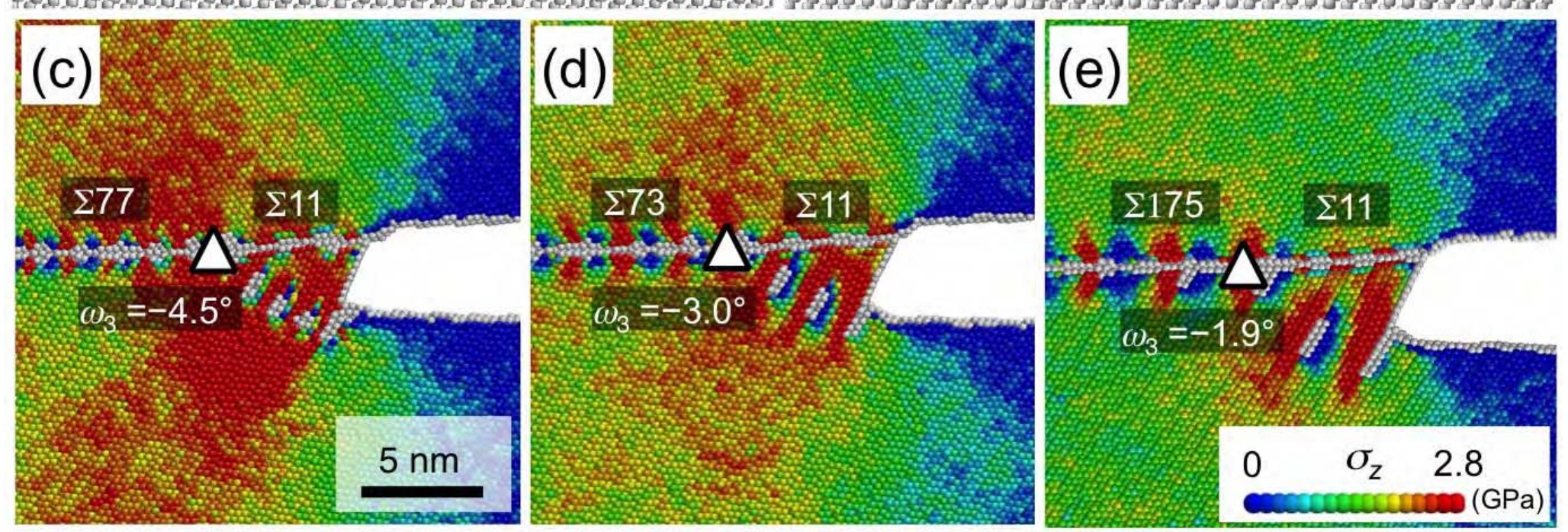
Figure 12
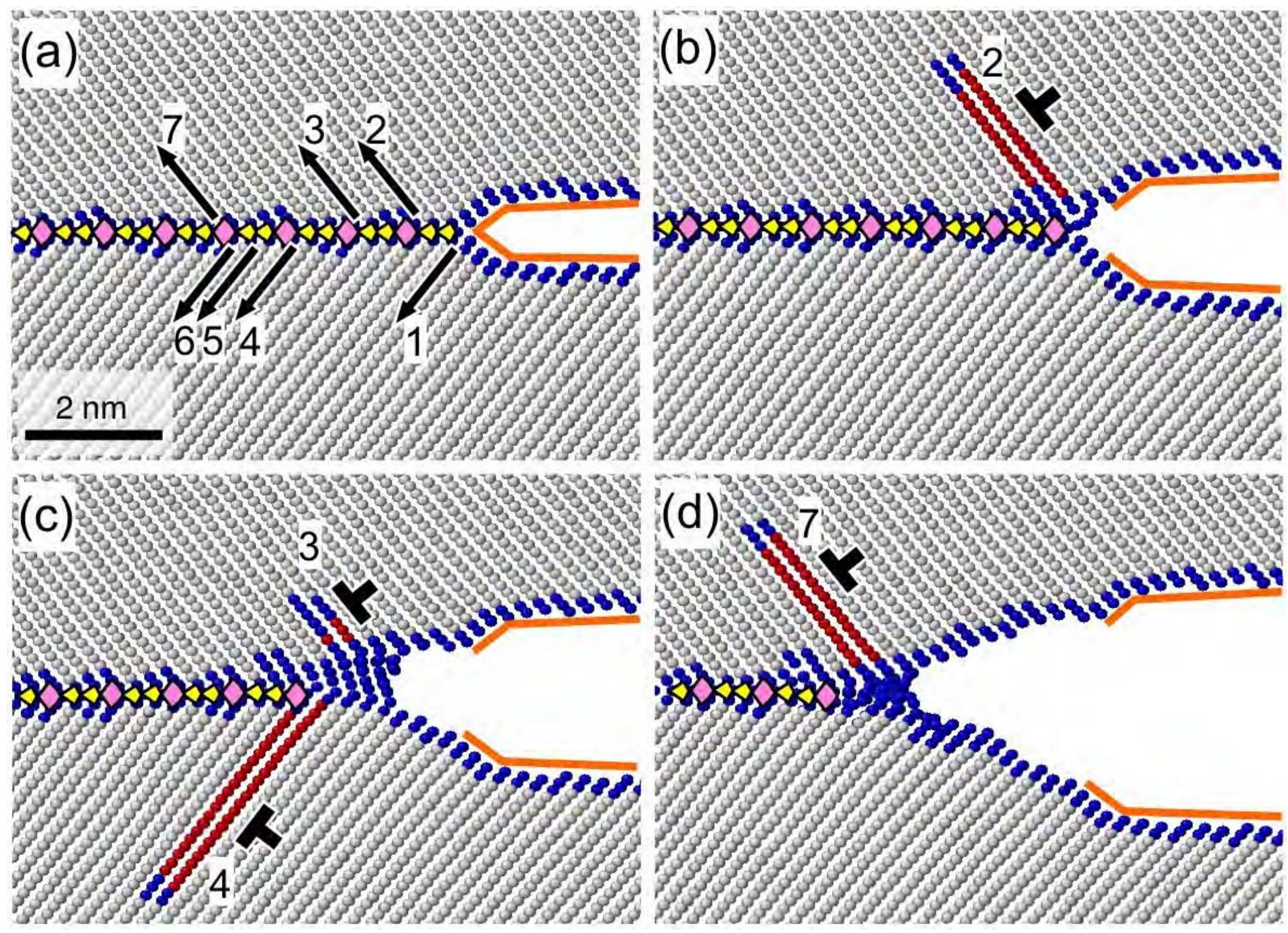
Figure B1
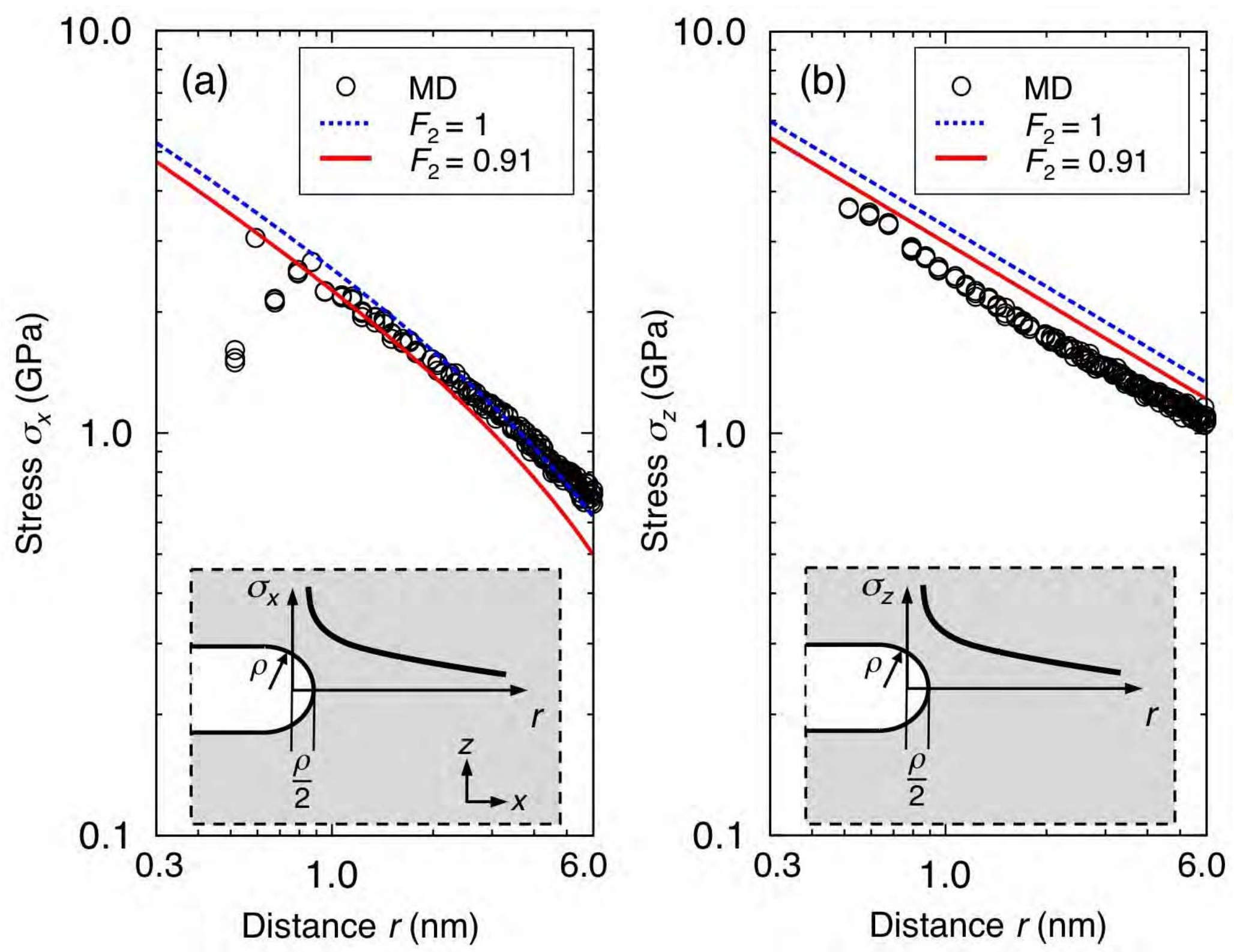
Figure C1

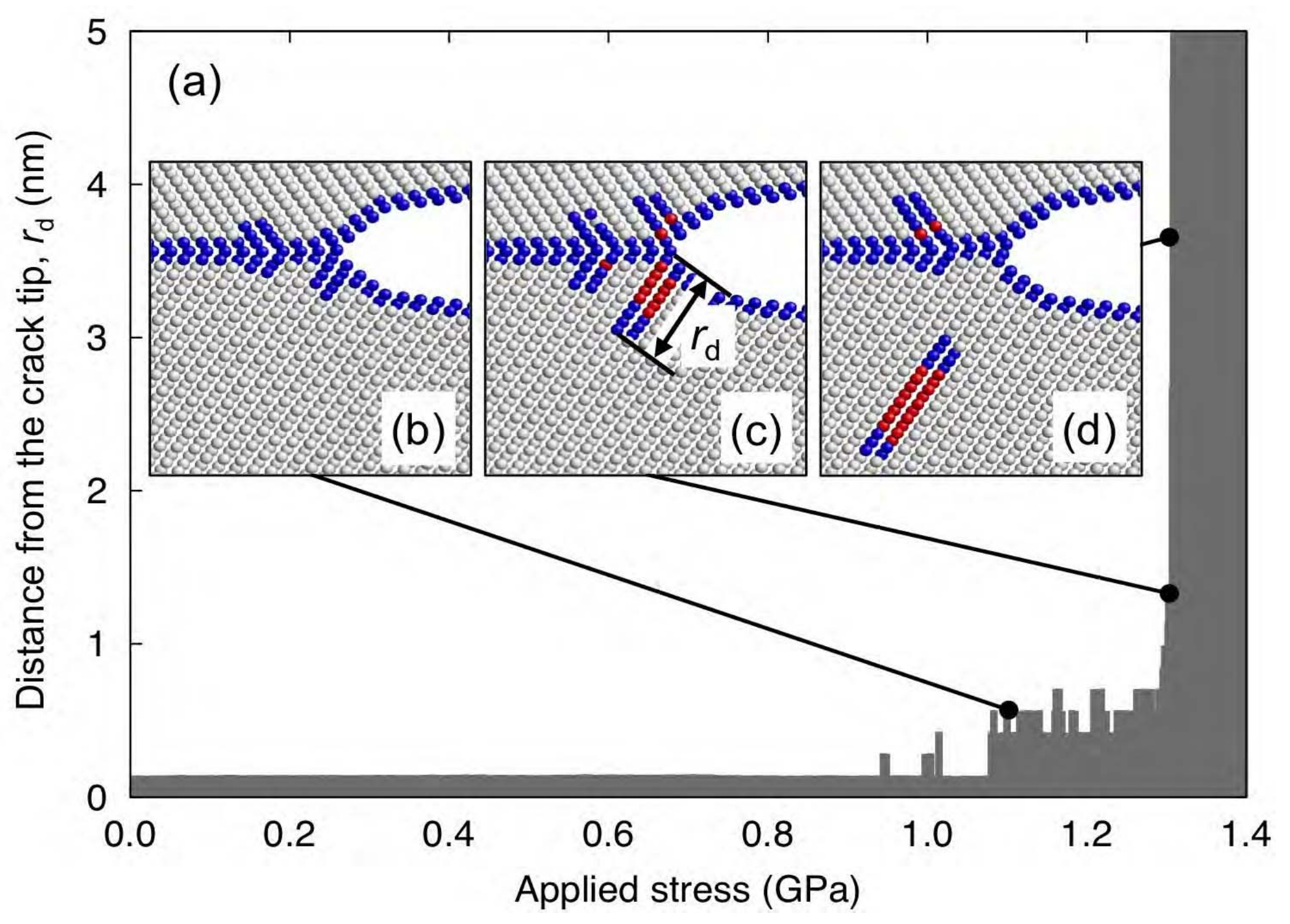

